\title{
ON EXTENSIONS FOR GENTLE ALGEBRAS
}

\author{
İLKE ÇANAKÇI, DAVID PAUKSZTELLO, AND SIBYLLE SCHROLL
}

\begin{abstract}
We give a complete description of a basis of the extension spaces between indecomposable string and quasi-simple band modules in the module category of a gentle algebra.
\end{abstract}

\section{INTRODUCTION}

The representation theory of finite-dimensional algebras plays an important role in many different areas of mathematics, such as in Lie theory, in number theory in connection with the Langlands program and automorphic forms, in geometry ranging from invariant theory to non-commutative resolutions of singularities and as far afield as harmonic analysis where the representation theory of $S^{1}$ appears in the guise of Fourier analysis.

Most finite-dimensional algebras are of wild representation type, that is their representation theory is at least as complicated as that of the free associative algebra in two generators. An algebra that is not wild is of tame representation type. One particular class of tame algebras, the so-called gentle algebras appear in a surprising number of different contexts. For example, in the context of Fukaya categories related to Kontsevich's homological mirror symmetry program [14, 21, 23], of dimer models [6], of the enveloping algebras of Lie algebras [16], and in the context of cluster theory as $(m$-)cluster tilted and $m$-Calabi Yau tilted algebras and also as Jacobian algebras associated to unpunctured surfaces $[2,4,13,20]$. Furthermore, the class of derived-discrete algebras consists of gentle algebras [27].

But there are many other reasons why gentle algebras have been studied extensively. One of the main reasons being that they are string algebras and that their indecomposable representations are classified by string and band modules [28], see also [8]. The associated string combinatorics governs the representation theory of gentle algebras, examples of this are the classification of morphisms between string and band modules [12, 19] and a characterisation of almost split sequences in terms of string combinatorics [8]. Over last few years, interest in gentle algebras has intensified with many new results appearing, an example of this is the recent work [24], where string combinatorics is used to classify support $\tau$-tilting modules.

Another reason for the extensive investigation of gentle algebras is the fact that they are derived tame and the indecomposable objects in the derived category of a gentle algebra have been classified. They are given by the so-called homotopy strings and bands [5]. In [1] the morphisms between string and band complexes in the derived category of a gentle algebra were characterised in terms of homotopy string combinatorics and in $[9,10]$ a graphical mapping cone calculus based on the morphisms described in [1] was developed.

Extensions between modules are one of the fundamental cohomological tools. Not only do they play an essential role in the definition of, for example, the Yoneda algebra or Hochschild cohomology, they are also essential in many of the newer developments in representation theory such as in cluster tilting in cluster theory.

The projective resolutions of indecomposable modules over gentle algebras are well understood, see, for example, [18]. So it is surprising that up to now, in general, no complete combinatorial description of the extensions between indecomposable modules over a gentle algebra is known. A description of certain combinatorially defined extensions between string modules

2010 Mathematics Subject Classification. 16G10, 16E35, 18E30, 05E10.

Key words and phrases. gentle algebra, extensions, bounded derived category, homotopy string and bands, string combinatorics . 
was given in [25], we will refer to these extensions as arrow and overlap extensions. In [29] it was shown that the existence of such extensions is a necessary and sufficient condition for the non-vanishing of the Ext ${ }^{1}$-space.

However, it has remained an open problem for almost twenty years whether these extensions form a basis of the $\mathrm{Ext}^{1}$-space between string modules and what the extensions involving band modules are. In fact, it has become apparent that string combinatorics in the module category of a gentle algebra might not be enough to answer this question. This has further been confirmed by the recent results in [11] where based on arguments using the associated cluster category, it was shown that in the context of gentle Jacobian algebras of quivers with potential, the extensions between string modules described in [25] do indeed give a basis.

In this paper, we answer this open question by giving, for any gentle algebra, a basis of the extension space between indecomposable modules. More precisely, we explicitly determine the cohomology of the indecomposable objects in the bounded derived category of a gentle algebra given in terms of homotopy strings and bands. Building on this we give a complete description of the extension space between string and quasi-simple band modules by giving a combinatorial description of a basis. We do this by working not in the module category of a gentle algebra, but we transfer the problem into the derived category, where we are able to use the graphical mapping cone calculus developed in $[9,10]$. We now state our main results; for the relevant definitions and details on notation, see Section 1 and Definition 3.1. Throughout the following, $\mathbf{k}$ will be an algebraically closed field and $\Lambda=\mathbf{k} Q / I$ will be a gentle algebra.

Theorem A. Let $\Lambda$ be a gentle algebra and $v$ and $w$ strings with $M(v)$ and $M(w)$ the corresponding string modules over $\Lambda$. The collection of arrow and overlap extensions of $M(v)$ by $M(w)$ form a basis of $\operatorname{Ext}_{\Lambda}^{1}(M(v), M(w))$.

In the following we give a graphical presentation of the strings in an arrow and overlap extension.

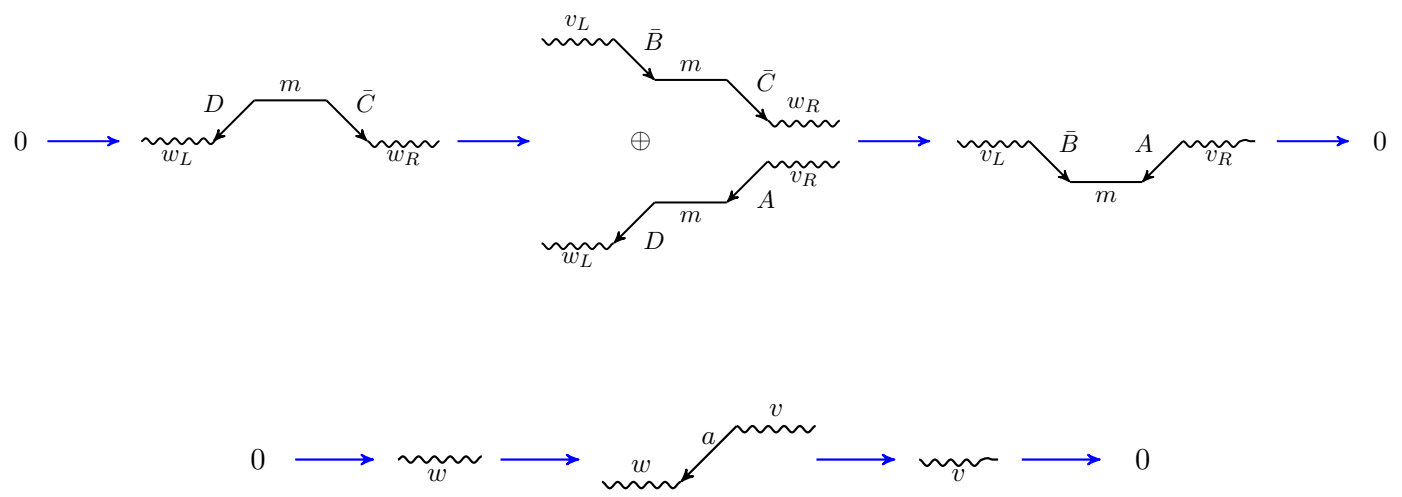

Figure 1. Presentation in terms of strings of an overlap extension (top picture) and an arrow extension (bottom picture) where for an arrow $a \in Q_{1}$ we denote its formal inverse by $\bar{a}$.

We note that in concurrent work [7], which builds on [22], a basis for extensions between string modules over a gentle algebra is also given using different techniques.

When a band is involved there are no arrow extensions, only overlap extensions. An extension involving both a string module and a band module has only one indecomposable module as its middle term. An extensions involving two band modules can have as its middle term the direct sum of many indecomposable band modules. The following theorems describe the situation involving band modules more precisely. Given a band $b$ and a scalar $\mu \in \mathbf{k}^{*}$, we denote the associated quasi-simple band module by $B(b, \mu)$. A useful comparison for the following statements is the corresponding statements for mapping cones of quasi-graph maps involving a band complex given in [10]. In the following, for a band $b$, denote by ${ }^{\infty} b^{\infty}$ (resp. ${ }^{\infty} b$, resp. $b^{\infty}$ ) 
the string obtained from $b$ by repeatedly concatenating $b$ with itself both on the left and on the right (resp. on the left, resp. on the right).

Theorem B. Let $\Lambda$ be a gentle algebra, $v$ be a string and $(b, \mu)$ be a band with $\mu \in \mathbf{k}^{*}$. Suppose that $v$ and ${ }^{\infty} b^{\infty}$ admit decompositions

$$
v=v_{L} \bar{B} m A v_{R} \text { and }{ }^{\infty} b^{\infty}={ }^{\infty} b b_{L} \operatorname{Dm} \bar{C} b_{R} b^{\infty},
$$

where $A, B, C, D \in Q_{1}$ with $C \neq \varnothing \neq D$ and $v_{L}, v_{R}, m, b_{L}$ and $b_{R}$ are (possibly trivial) strings satisfying the conditions of Definition 3.1(2).

(a) If, after suitable rotation of $b, m$ is a proper subword of $b$, then there is a non-split overlap extension

$$
0 \rightarrow B(b, \mu) \rightarrow M(u) \rightarrow M(v) \rightarrow 0,
$$

where $u=v_{L} \bar{B} m \bar{C} b_{R} b_{L} D m A v_{R}$ is a string.

(b) If $b$ is a subword of $m$, then after suitable rotation of $b$ there is a decomposition $b=b_{2} b_{1}$ such that $m=b^{k} b_{2}$ for some $k \geq 1$ and there is a non-split overlap extension

$$
0 \rightarrow B(b, \mu) \rightarrow M(u) \rightarrow M(v) \rightarrow 0,
$$

where $u=v_{L} \bar{B} b^{k+1} b_{2} A v_{R}$ is a string.

Moreover, the collection of such extensions forms a basis of $\operatorname{Ext}_{\Lambda}^{1}(M(v), B(b, \mu))$.

Theorem C. Let $\Lambda$ be a gentle algebra, $(c, \lambda)$ be a band with $\lambda \in \mathbf{k}^{*}$ and $w$ be a string. Suppose that ${ }^{\infty} c^{\infty}$ and $w$ admit decompositions

$$
\infty_{c}^{\infty}={ }^{\infty} c c_{L} \bar{B} m A c_{R} c^{\infty} \text { and } w=w_{L} D m \bar{C} w_{R},
$$

where $A, B, C, D \in Q_{1}$ with $A \neq \varnothing \neq B$ and $c_{L}, c_{R}, m, w_{L}$ and $w_{R}$ are (possibly trivial) strings satisfying the conditions of Definition 3.1(2).

(a) If, after suitable rotation of $c, m$ is a proper subword of $c$, then there is a non-split overlap extension

$$
0 \rightarrow M(w) \rightarrow M(u) \rightarrow B(c, \lambda) \rightarrow 0,
$$

where $u=w_{L} D m A c_{R} c_{L} \bar{B} m \bar{C} w_{R}$ is a string.

(b) If $c$ is a subword of $m$, then after suitable rotation of $c$ there is a decomposition $c=c_{2} c_{1}$ such that $m=c^{\ell} c_{2}$ for some $\ell \geq 1$ and there is a non-split overlap extension

$$
0 \rightarrow M(w) \rightarrow M(u) \rightarrow B(c, \lambda) \rightarrow 0,
$$

where $u=w_{L} D c^{\ell+1} c_{2} \bar{C} w_{R}$ is a string.

Moreover, the collection of such extensions forms a basis of $\operatorname{Ext}_{\Lambda}^{1}(B(c, \lambda), M(w))$.

Theorem D. Let $\Lambda$ be a gentle algebra and $(b, \mu) \neq(c, \lambda)$ be bands with $\lambda, \mu \in \mathbf{k}^{*}$. Suppose that ${ }^{\infty} c^{\infty}$ and ${ }^{\infty} b^{\infty}$ admit decompositions

$$
{ }^{\infty} c^{\infty}={ }^{\infty} c_{L} \bar{B} m A c_{R} c^{\infty} \text { and }{ }^{\infty} b^{\infty}={ }^{\infty} b b_{L} D m \bar{C} b_{R} b^{\infty},
$$

where $A, B, C, D \in Q_{1}$ are each nonempty and $c_{L}, c_{R}, m, b_{L}$ and $b_{R}$ are (possibly trivial) strings satisfying the conditions of Definition 3.1(2). Then, either

(a) $m$ is a proper subword of $b$, i.e. after suitable rotation of $b$ there is a decomposition $b=m v ;$ or,

(b) $b$ is a subword of $m$, i.e. after suitable rotation there is a decomposition $b=b_{2} b_{1}$ such that $m=b^{k} b_{2}$,

and, either,

(c) $m$ is a proper subword of $c$, i.e. after suitable rotation of $c$ there is a decomposition $c=m w ;$ or

(d) $c$ is a subword of $m$, i.e. after suitable rotation there is a decomposition $c=c_{2} c_{1}$ such that $m=c^{\ell} c_{2}$. 
Then, there is a band $d$ and an integer $t \geq 1$ such that

$$
d^{t}= \begin{cases}m v m w & \text { if (a) } \mathscr{E}(c) ; \\ m v c_{2} c_{1} & \text { if }(a) \mathscr{E}(d) ; \\ b_{2} b_{1} m w & \text { if }(b) \mathscr{E}(c) ; \\ b_{2} b_{1} c_{2} c_{1} & \text { if }(b) \mathscr{E}(d),\end{cases}
$$

and a non-split overlap extension

$$
0 \rightarrow B(b, \mu) \rightarrow \bigoplus_{i=1}^{t} B\left(d, \omega^{i} \sqrt[t]{ \pm \lambda \mu^{-1}}\right) \rightarrow B(c, \lambda) \rightarrow 0,
$$

where $\omega$ is a primitive $t^{\text {th }}$ root of unity. Moreover, the collection of such extensions forms a basis of $\operatorname{Ext}_{\Lambda}^{1}(B(c, \lambda), B(b, \mu))$.

Theorem E. Let $\Lambda$ be a gentle algebra and $(b, \mu)$ be a band with $\mu \in \mathbf{k}^{*}$. The collection of extensions in Theorem $D$ in which $m \neq b$ together with the Auslander-Reiten sequence,

$$
0 \rightarrow B(b, \mu) \rightarrow B\left(b, \mu, \mathbf{k}^{2}\right) \rightarrow B(b, \mu) \rightarrow 0,
$$

where $B\left(b, \mu, \mathbf{k}^{2}\right)$ denotes the 2-dimensional band module with Jordan block whose eigenvalue is $\mu$, form a basis of $\operatorname{Ext}_{\Lambda}^{1}(B(b, \mu), B(b, \mu))$.

Remark. In Theorem D, each of the words defining $d^{t}$ is, after suitable rotation of $b$ and $c$ just the concatenation of the two bands, $b c$. However, different possibilities for $d$ arise from the precise decompositions of $b$ and $c$ : for different $m$, concatenations $b c$ with respect to different decompositions need not be equivalent up to inverting the word or cyclic permutation.

We now briefly outline the content of the paper, including the general strategy of the proofs of Theorems A, B, C, D and E. Let $\Lambda$ be a gentle algebra. We begin by recalling the basic notions of string and homotopy string combinatorics for gentle algebras in Section 1. In Section 2 we determine the homotopy string or band of the minimal projective resolution of a string or band module over $\Lambda$ and the cohomology of a string or band complex in $\mathrm{K}^{b,-}(\operatorname{proj}(\Lambda))$.

In order to describe the content of Sections 3 and 4 more precisely, fix the following notation. Let $v$ and $w$ be strings or bands and $M(v)$ and $M(w)$ the corresponding string or quasi-simple band modules. We denote the homotopy strings or bands of their projective resolutions by $\pi(v)$ and $\pi(w)$ and the corresponding string or band complexes by $Q_{\pi(v)}^{\bullet}$ and $Q_{\pi(w)}^{\bullet}$. The standard basis of homomorphisms between string and/or band complexes is recalled from [1] in Section 1.4, enabling us to give an explicit description of a basis of $\operatorname{Hom}_{K^{b,-}(\operatorname{proj}(\Lambda))}\left(Q_{\pi(v)}^{\bullet}, \Sigma Q_{\pi(w)}^{\bullet}\right)$.

In the first step in the proof, we show in Section 3 that the image of every element of the standard basis under the canonical isomorphism

$$
\Phi: \operatorname{Hom}_{\mathrm{K}^{b,-}(\operatorname{proj}(\Lambda))}\left(Q_{\pi(v)}^{\bullet}, \Sigma Q_{\pi(w)}^{\bullet}\right) \stackrel{\sim}{\rightarrow} \operatorname{Ext}_{\Lambda}^{1}(M(v), M(w))
$$

is either an overlap or an arrow extension. In particular, this shows that the set of overlap and arrow extensions form a generating set for $\operatorname{Ext}_{\Lambda}^{1}(M(v), M(w))$.

The second step of the proof, comprising Section 4, shows that the set of overlap and arrow extensions forms a basis of $\operatorname{Ext}_{\Lambda}^{1}(M(v), M(w))$. To see this, we show that $\Phi$ restricts to a surjection from the standard basis of $\operatorname{Hom}_{\mathrm{K}^{b,-}(\operatorname{proj}(\Lambda))}\left(Q_{\pi(v)}^{\bullet}, \Sigma Q_{\pi(w)}^{\bullet}\right)$ to the set of arrow and overlap extensions in $\operatorname{Ext}_{\Lambda}^{1}(M(v), M(w))$.

We emphasise that, with the exception of the case highlighted in the remark above, the methods apply equally to (homotopy or classical) strings and bands. Furthermore, for ease of the already somewhat heavy notation, in the proofs in Section 3 and 4, whenever we have a map between two band complexes or an extension between two band modules, implicitly and without loss of generality we assume that the parameters of the corresponding band complexes or band modules are equal to one, see $[9, \S 2.3]$ for more details on the placement of parameters with respect to mapping cones. 
Acknowledgments. The second author would like to thank Raquel Coelho Simões and Rosanna Laking for useful comments and corrections. The authors would also like to thank an anonymous referee for a thorough reading of the article and many useful comments that have significantly improved the exposition. This work has been supported by the EPSRC through the grants EP/K026364/1, EP/K022490/1 and EP/N005457/1. The third author is supported by the EPSRC through an Early Career Fellowship EP/P016294/1.

\section{BACKGROUND}

In this section we briefly recall the definition of gentle algebras, background on string and band modules, string and band complexes and the standard basis of the morphism spaces between string and band complexes that will be needed in the article.

1.1. Gentle algebras. Throughout, $\mathbf{k}$ will be an algebraically closed field. We recall the following definition from [3].

Definition 1.1. A finite-dimensional $\mathbf{k}$-algebra $\Lambda$ is gentle if it is Morita equivalent to a bound path algebra $\mathrm{k} Q / I$, where $Q$ is a quiver and $I$ an admissible ideal in $\mathbf{k} Q$ such that

(1) for each vertex $i \in Q_{0}$ there are at most two arrows starting at $i$ and at most two arrows ending at $i$;

(2) for each arrow $a \in Q_{1}$ there is at most one arrow $b$ with $e(a)=s(b)$ and such that $b a \notin I$ and at most one arrow $c$ with $e(c)=s(a)$ and such that $a c \notin I$;

(3) for each arrow $a \in Q_{1}$ there is at most one arrow $b$ with $e(a)=s(b)$ and such that $b a \in I$ and at most one arrow $c$ with $e(c)=s(a)$ and such that $a c \in I$;

(4) the ideal $I$ is generated by length-two monomial relations.

From now on $\Lambda=\mathbf{k} Q / I$ will be a gentle algebra.

1.2. String and band modules. We now describe strings and bands, which parametrise the indecomposable $\Lambda$-modules. The reference for this material is $[8,28]$. Note that, in this paper all modules will be finitely generated left modules, and therefore paths in the quiver will be read from right to left.

For each arrow $a \in Q_{1}$ we introduce a formal inverse arrow $\bar{a}=a^{-1}$ with $s(\bar{a})=e(a)$ and $e(\bar{a})=s(a)$. We write $\bar{Q}_{1}$ for the set of formal inverse arrows. Similarly for a path $p=a_{n} \cdots a_{1}$ the inverse path is $\bar{p}=\bar{a}_{1} \cdots \bar{a}_{n}$. Sometimes we shall assert the nonexistence of an arrow or inverse arrow $a$, and in this case we write $a=\varnothing$.

Definitions 1.2. We recall the following notions.

(1) A walk of length $l>0$ in $(Q, I)$ is a sequence $w=w_{l} \cdots w_{1}$ satisfying $s\left(w_{i+1}\right)=e\left(w_{i}\right)$, where each $w_{i}$ is either an arrow or an inverse arrow, and where the sequence does not contain any subsequence of the form $a \bar{a}$ or $\bar{a} a$ for an arrow $a \in Q_{1}$. We will call each arrow or inverse arrow $w_{i}$ in $w$ a letter of $w$.

(2) A string is a walk that does not contain subwalks $v$ such that $v \in I$ or $\bar{v} \in I$. In addition, there are trivial strings $1_{x}$ for each vertex $x \in Q_{0}$.

(3) A band is a string $b=b_{n} \cdots b_{1}$ such that $e\left(b_{n}\right)=s\left(b_{1}\right), b_{1} \neq \overline{b_{n}}, b_{1} b_{n}$ is defined as a string, and $b \neq v^{m}$ for some substring $v$ and $m>1$.

Modulo the equivalence relation $w \sim \bar{w}$ the strings form an indexing set for the so-called string modules. Given a string $w$, we write $M(w)$ for the corresponding string module. Note that if $w=1_{x}$ is a trivial string $M(w)=S(x)$ is the simple module at $x$. We refer to $[8,28]$ for more details on how to construct string modules from strings.

Modulo the equivalence relation given by inversion and cyclic permutation (rotation), the bands together with scalars $\mu \in \mathbf{k}^{*}$ form an indexing set for the so-called band modules, $B(b, \mu)$, where by convention we place $\mu$ on a direct arrow. By abuse of notation, we will usually drop the scalar and write simply $B(b)$ for the corresponding band module. Again we refer to [8] for the actual construction of the band modules. 
In order to deal with the word combinatorics involving bands effectively, we will need to consider infinite periodic words corresponding to bands. Let $b$ a band, we write

$$
\begin{aligned}
\infty^{\infty} & =\cdots \underbrace{b_{n} \cdots b_{1}}_{b} \underbrace{b_{n} \cdots b_{1}}_{b} \underbrace{b_{n} \cdots b_{1}}_{b} \cdots, \\
\infty_{b} & =\cdots \underbrace{b_{n} \cdots b_{1}}_{b} \underbrace{b_{n} \cdots b_{1}}_{b}, \quad \text { and }, \\
b^{\infty} & =\underbrace{b_{n} \cdots b_{1}}_{b} \underbrace{b_{n} \cdots b_{1}}_{b} \cdots .
\end{aligned}
$$

In particular, let $(b, \mu)$ and $(c, \lambda)$ be bands, then $(b, \mu)=(c, \lambda)$ if and only if ${ }^{\infty} b^{\infty}={ }^{\infty} c^{\infty}$ or $\infty b^{\infty}={ }^{\infty}\left(c^{-1}\right)^{\infty}$ and $\lambda=\mu$ with both $\lambda$ and $\mu$ placed on a direct arrow in the infinite words that are equal.

By [28, Prop. 2.3], the string and band modules form a complete set of isomorphism classes of indecomposable $\Lambda$-modules.

The band modules given by representations in which each vertex is replaced by a 1dimensional vector space all lie at the mouth of homogeneous tubes and are referred to as quasi-simple (band) modules. They can be characterised as those band modules $B$ such that there exists an almost split sequence of the form $0 \rightarrow B \rightarrow E \rightarrow \tau^{-1} B \rightarrow 0$ where $E$ is indecomposable, see for example [26]. In the following by abuse of notation, whenever we will use the term band module we will be referring to a quasi-simple band module.

1.3. String and band complexes. We now describe homotopy strings and bands, which parametrise the indecomposable complexes in the derived category $\mathrm{D}^{b}(\Lambda)$. We will use the notation and terminology employed in $[1,9]$ and the references therein. However, for the sake of brevity we drop some of the formality of $[1,9]$ regarding the degrees.

Definitions 1.3. The original reference for the following definitions is [5].

(1) A (finite) homotopy string is a walk of finite length in $(Q, I)$. In addition, there are trivial homotopy strings for each vertex $x \in Q_{0}$.

(2) A subwalk $p=w_{j} \cdots w_{i}$ of a homotopy string $\sigma=w_{l} \cdots w_{1}$ is a homotopy letter if

(a) $p$ or $\bar{p}$ is a path of length at least one in $(Q, I)$; and,

(b) $w_{i} \in Q_{1}$ and $w_{i-1} \in \bar{Q}_{1}$ or vice versa, or $w_{i} w_{i-1} \in I$, or $\overline{w_{i-1} w_{i}} \in I$; and,

(c) $w_{j} \in Q_{1}$ and $w_{j+1} \in \bar{Q}_{1}$ or vice versa, or $w_{j+1} w_{j} \in I$, or $\overline{w_{j} w_{j+1}} \in I$.

We say that $p$ is a direct homotopy letter if it is a path in $(Q, I)$ and an inverse homotopy letter if $\bar{p}$ is a path in $(Q, I)$. In this way we partition a homotopy string $\sigma$ into homotopy letters and write $\sigma=\sigma_{n} \cdots \sigma_{1}$ for this decomposition. A homotopy subletter of $p$ is a subwalk of $p$ of length at least one.

(3) A homotopy letter $p=w_{l} \cdots w_{1}$, with $w_{i} \in Q_{1}$ for $i=1, \ldots, l$ or $\bar{w}_{i} \in Q_{1}$ for $i=1, \ldots, l$, is said to have length $l$ and we write $\operatorname{length}(p)=l$. The length can be zero, in which case $p=1_{x}$ for some $x \in Q_{0}$ and $p$ is called a trivial homotopy letter. Sometimes we shall assert the nonexistence of homotopy letters, and in this case we write $p=\varnothing$.

(4) Let $\sigma=\sigma_{n} \cdots \sigma_{1}$ be a homotopy string decomposed into its homotopy letters. A subwalk $\tau=\sigma_{j} \cdots \sigma_{i}$ with $1 \leq i \leq j \leq n$ is called a homotopy substring of $\sigma$.

(5) A homotopy band is a homotopy string $\sigma=\sigma_{n} \cdots \sigma_{1}$ with $s(\sigma)=e(\sigma), \sigma_{1} \neq \bar{\sigma}_{n}, \sigma \neq \tau^{m}$ for some homotopy substring $\tau$ and $m>1$, and $\sigma$ has equal numbers of direct and inverse homotopy letters.

Remark 1.4. Throughout the article, whenever we write a walk using Greek letters, such as $\sigma=\sigma_{n} \cdots \sigma_{1}$, we will always mean its decomposition into homotopy letters whereas, in general, we reserve Roman letters for (classical) strings and bands.

Modulo the equivalence relation $\sigma \sim \bar{\sigma}$ the homotopy strings form an indexing set for the so-called string complexes. Given a homotopy string $\sigma$, we write $P_{\sigma}^{\bullet}$ for the corresponding string complex. Note that if $\sigma=1_{x}$ is a trivial homotopy string $P_{\sigma}^{\bullet}=P(x)$ is the stalk complex of the 
projective module at $x$. We refer to $[1,5]$ for more details on how to construct string complexes from homotopy strings; for a sketch of the constructions, see Example 1.5 below.

Modulo the equivalence relation given by inversion and cyclic permutation, the homotopy bands together with scalars $\lambda \in \mathbf{k}^{*}$ form an indexing set for the so-called band complexes $B_{\sigma, \lambda}^{\bullet}$. Again we refer to $[1,5]$ for the actual construction of the band complexes.

By [5, Thm. 3], the string and band complexes form a complete set of indecomposable perfect complexes in $\mathrm{D}^{b}(\Lambda)$. For the remaining objects of $\mathrm{D}^{b}(\Lambda)$ we need some further terminology.

Example 1.5 ([1, Running Example]). Let $\Lambda=\mathbf{k} Q / I$ be given by the following bound quiver:

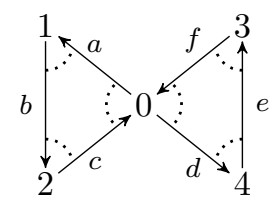

Consider the following indecomposable complex in $\mathrm{D}^{b}(\Lambda)$, where we assume the left-most nonzero term is in cohomological degree zero.

$$
0 \longrightarrow P(0) \stackrel{[c f]}{\longrightarrow} P(2) \oplus P(3) \stackrel{\left[\begin{array}{ll}
b & 0 \\
0 & e
\end{array}\right]}{\longrightarrow} P(1) \oplus P(4) \stackrel{\left[\begin{array}{c}
a f \\
0
\end{array}\right]}{\longrightarrow} P(3) \longrightarrow 0 .
$$

This complex can be 'unfolded' to give the following diagram,

$$
P(4) \stackrel{\bar{e}}{\longleftarrow} P(3)<\frac{\bar{f}}{\longleftarrow} P(0) \stackrel{c}{\longrightarrow} P(2) \stackrel{b}{\longrightarrow} P(1) \stackrel{\text { af }}{\longrightarrow} P(3) .
$$

The indecomposable projective modules appearing are uniquely determined by the endpoints of the maps, so all information in this complex is contained in the diagram



Here the homotopy string $\sigma=\bar{e} \bar{f} c b a f$, and we refer to (2) as the 'unfolded diagram' of $\sigma$. For more details we refer the reader to $[1, \S 2]$.

Definitions 1.6. In the following, walks may now be infinite (on both sides).

(1) A walk $w$ is called a direct antipath if it is direct and in its decomposition into homotopy letters, each homotopy letter has length 1 ; it is called an inverse antipath if it is inverse and in its decomposition into homotopy letters, each homotopy letter has length 1.

(2) A left infinite walk $w=\cdots w_{n} \cdots w_{2} w_{1}$ is a left infinite homotopy string if there exists $m \geq 1$ such that $v=\cdots w_{n} \cdots w_{m+1} w_{m}$ is a direct antipath.

(3) A right infinite walk $w=w_{-1} w_{-2} \cdots w_{-n} \cdots$ is a right infinite homotopy string if there exists $m \geq 1$ such that $v=w_{-m} w_{-m-1} \cdots w_{-n} \cdots$ is an inverse antipath.

(4) A two sided infinite walk $w=\cdots w_{2} w_{1} w_{0} w_{-1} \cdots$ is called a two-sided infinite homotopy string if there exist integers $n>m$ such that $\cdots v_{n+1} v_{n}$ is a direct antipath and $v_{m} v_{m-1} \cdots$ is an inverse antipath.

(5) By a one-sided infinite homotopy string we mean either a left infinite homotopy string or a right infinite homotopy string.

By [5, Thm. 3] the indecomposable non-perfect complexes in $\mathrm{D}^{b}(\Lambda)$ are parametrised by the one-sided and two-sided infinite homotopy strings; they are again called string complexes. In the following, we write

$$
Q_{\sigma}^{\bullet}= \begin{cases}P_{\sigma}^{\bullet} & \text { if } \sigma \text { is a (possibly infinite) homotopy string; } \\ B_{\sigma, \lambda}^{\bullet} & \text { if } \sigma \text { is a homotopy band. }\end{cases}
$$

From now on, by abuse of terminology, we say homotopy string for a (possibly infinite) homotopy string. 
1.4. The standard basis. A basis for the morphism space between indecomposable complexes in $\mathrm{D}^{b}(\Lambda)$ was determined in [1]. Here we briefly recall this basis, which we shall refer to as the standard basis. As observed in Example 1.5, homotopy strings and bands correspond to an unfolding of the corresponding string and band complexes. Throughout the paper, we shall freely make use of the unfolded diagram notation for string and band complexes from $[1,9]$.

Theorem 1.7 ([1, Theorem 3.15]). Let $\sigma$ and $\tau$ be homotopy strings or bands. Then there is a canonical basis of $\operatorname{Hom}_{\mathrm{D}^{b}(\Lambda)}\left(Q_{\sigma}^{\bullet}, Q_{\tau}^{\bullet}\right)$ given by:

- graph maps $f^{\bullet}: Q_{\sigma}^{\bullet} \rightarrow Q_{\tau}^{\bullet}$;

- singleton single maps $f^{\bullet}: Q_{\sigma}^{\bullet} \rightarrow Q_{\tau}^{\bullet}$;

- singleton double maps $f^{\bullet}: Q_{\sigma}^{\bullet} \rightarrow Q_{\tau}^{\bullet}$;

- quasi-graph maps $\varphi: Q_{\sigma}^{\bullet} \rightsquigarrow \Sigma^{-1} Q_{\tau}^{\bullet}$.

We note that a quasi-graph map is not a map, but in fact determines classes of homotopy equivalent single and double maps, which is why we denote it by $\rightsquigarrow$ and not $\rightarrow$.

Throughout the following description of the maps listed above, $\sigma$ and $\tau$ will be homotopy strings or bands.

1.4.1. Graph maps. Suppose $\sigma$ and $\tau$ are, up to inversion, of the form,

(1) $\sigma=\beta \sigma_{L} \rho \sigma_{R} \alpha$ and $\tau=\delta \tau_{L} \rho \tau_{R} \gamma$; or

(2) $\sigma=\rho \sigma_{R} \alpha$ and $\tau=\rho \tau_{R} \gamma$,

where $\alpha, \beta, \gamma$ and $\delta$ are homotopy substrings, $\sigma_{L}, \sigma_{R}, \tau_{L}$ and $\tau_{R}$ are (possibly trivial) homotopy letters, and $\rho$ is a (possibly trivial) maximal common homotopy substring, and in the second case an infinite homotopy substring of $\sigma$ and $\tau$. We assume that $\rho$ occurs in the same cohomological degrees in both homotopy strings. Then the corresponding graph maps can be represented by the following unfolded diagrams:
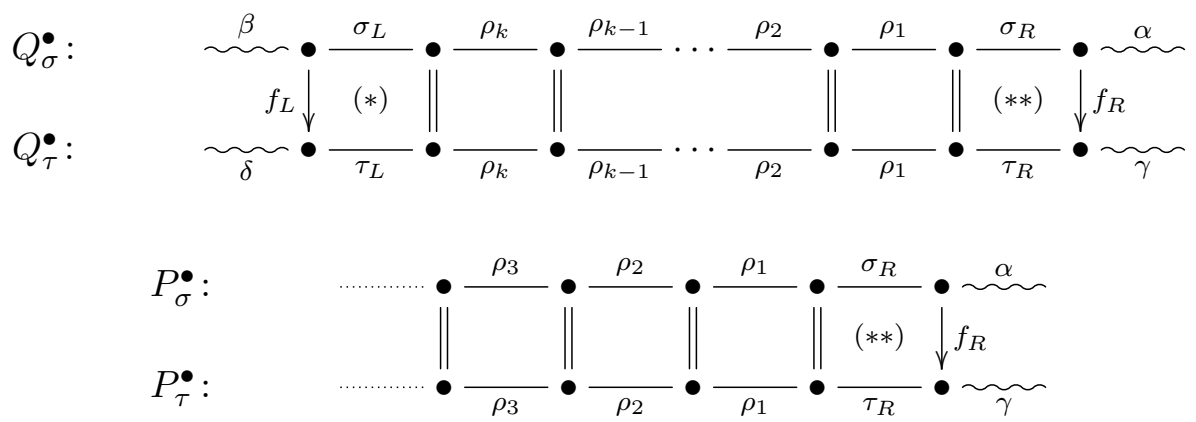

where we require the squares marked $(*)$ and $(* *)$ to commute; these are explicitly written down in $[1, \S 3.2]$. The maximality of $\rho$ as a common homotopy substring of $\sigma$ and $\tau$ necessarily means that $\sigma_{L} \neq \tau_{L}$ and $\sigma_{R} \neq \tau_{R}$. Note that in the case of 1.4.1(2), $\rho$ is an antipath and we say that the graph map $f^{\bullet}$ is incident with $\rho$.

1.4.2. Single maps. The unfolded diagram of a single map $f^{\bullet}: Q_{\sigma}^{\bullet} \rightarrow Q_{\tau}^{\bullet}$ is given by

$$
\begin{gathered}
Q_{\sigma}^{\bullet}: \\
f^{\bullet} \downarrow \\
Q_{\tau}^{\bullet}:
\end{gathered}
$$

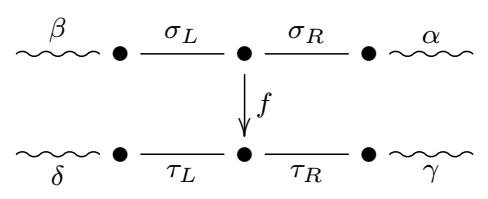

where $f$ is a nontrivial path in $(Q, I)$, and satisfying the following conditions:

(L1) if $\sigma_{L} \neq \varnothing$ then $\sigma_{L}$ is either inverse or is direct and $\sigma_{L} f$ has a subpath in $I$.

(L2) if $\tau_{L} \neq \varnothing$ then $\tau_{L}$ is either direct or is inverse and $f \bar{\tau}_{L}$ has a subpath in $I$.

(R1) if $\sigma_{R} \neq \varnothing$ then $\sigma_{R}$ is either direct or is inverse and $\bar{\sigma}_{R} f$ has a subpath in $I$.

(R2) if $\tau_{R} \neq \varnothing$ then $\tau_{R}$ is either inverse or is direct and $f \tau_{R}$ has a subpath in $I$. 
A single map $f^{\bullet}: Q_{\sigma}^{\bullet} \rightarrow Q_{\tau}^{\bullet}$ is called a singleton single map if its unfolded diagram, up to inversion of one of the homotopy strings/bands, is



where $\sigma_{L}$ and $\tau_{L}$ never contain $f$ as a subletter, and whenever $\sigma_{L}$ is inverse or $\tau_{L}$ is direct, $f$ does not contain $\sigma_{L}$ or $\tau_{L}$ as a subletter, and any of $\sigma_{L}, \sigma_{R}, \tau_{L}$ and $\tau_{R}$ are permitted to be the empty homotopy letter $\varnothing$.

1.4.3. Double maps. The unfolded diagram of a double map $f^{\bullet}: Q_{\sigma}^{\bullet} \rightarrow Q_{\tau}^{\bullet}$ is

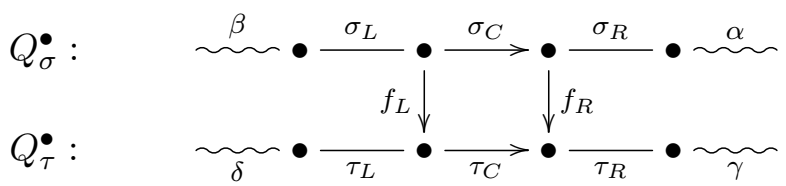

where $f_{L}$ and $f_{R}$ are nontrivial paths in $(Q, I)$ such that $f_{L} \tau_{C}=\sigma_{C} f_{R}$ has no subpath in $I$, conditions (L1) and (L2) hold for $f_{L}$ and (R1) and (R2) hold for $f_{R}$.

A double map, as above, is called singleton if there is a nontrivial path $f^{\prime}$ in $(Q, I)$ such that $\sigma_{C}=f_{L} f^{\prime}$ and $\tau_{C}=f^{\prime} f_{R}$.

1.4.4. Quasi-graph maps. If, in the situation of Section 1.4.1, the squares marked $(*)$ and $(* *)$ of diagrams (1) and (2) do not commute, then such diagrams determine a quasi-graph map $\varphi: Q_{\sigma}^{\bullet} \rightsquigarrow Q_{\tau}^{\bullet}$. The non-commuting endpoint conditions are explicitly spelled out in [9, §1.4.4]. Note that, while a quasi-graph map $Q_{\sigma}^{\bullet} \rightsquigarrow Q_{\tau}^{\bullet}$ does not define a map, a quasi-graph map $\varphi: Q_{\sigma}^{\bullet} \rightsquigarrow \Sigma^{-1} Q_{\tau}^{\bullet}$ determines a family of homotopy equivalent single and/or double maps. Indeed, all single and double maps that are not singleton arise in this way.

The following observation will be useful in the proofs in Section 4.

Remark 1.8. Suppose, in the unfolded diagram (1) above, $\rho_{1}$ is not the start of both $\sigma$ and $\tau$ and $\rho_{k}$ is not the end of both $\sigma$ and $\tau$. In this case, the diagram defines a graph map $f^{\bullet}: Q_{\sigma}^{\bullet} \rightarrow Q_{\tau}^{\bullet}$ if and only if the same diagram, when read upside down, i.e. from bottom to top, defines a quasi-graph map $\varphi: Q_{\tau}^{\bullet} \rightsquigarrow Q_{\sigma}^{\bullet}$. Note that we do not read $f_{L}$ and $f_{R}$ upside down: they occur as homotopy subletters of $\sigma_{L}$ or $\tau_{L}$ (resp. $\sigma_{R}$ or $\tau_{R}$ ). For example, consider the following case:



Here the fact that $\tau_{R}=\sigma_{R} f_{R}$ is the obstacle to the commuting of the right endpoint when the 'graph map' is read upside down, giving a (non-null-homotopic) quasi-graph map endpoint condition; see $[9, \S 1.4 .4]$.

1.5. Morphisms vs. extensions. For background on derived and homotopy categories we refer to [15]. One of the powerful features of the derived category is that it reformulates extensions in the module category in terms of morphisms. In particular, for any algebra $\Lambda$, and any $\Lambda$-modules $M$ and $N$ we have

$$
\begin{array}{ccccc}
\operatorname{Hom}_{\mathrm{K}}\left(P_{M}^{\bullet}, \Sigma P_{N}^{\bullet}\right) & \simeq & \operatorname{Ext}_{\mathrm{K}}^{1}\left(P_{M}^{\bullet}, P_{N}^{\bullet}\right) & \simeq & \operatorname{Ext}_{\Lambda}^{1}(M, N), \\
P_{M}^{\bullet} \stackrel{f^{\bullet}}{\longrightarrow} \Sigma P_{N}^{\bullet} & \mapsto & P_{N}^{\bullet} \longrightarrow C_{f^{\bullet}}^{\bullet} \longrightarrow P_{M}^{\bullet} \stackrel{f^{\bullet}}{\longrightarrow} \Sigma P_{N}^{\bullet} & \mapsto & 0 \rightarrow N \rightarrow H^{0}\left(C_{f^{\bullet}}^{\bullet}\right) \rightarrow M \rightarrow 0
\end{array}
$$

where $\mathrm{K}=\mathrm{K}^{b,-}(\operatorname{proj}(\Lambda)), P_{M}^{\bullet}$ and $P_{N}^{\bullet}$ are projective resolutions of $M$ and $N$, respectively, and $C_{f}^{\bullet}$ is the (negative shift of the) mapping cone of $f^{\bullet}$. In particular, computation of a 
basis of the Ext-space $\operatorname{Ext}_{\Lambda}^{1}(M, N)$ reduces to the computation of a basis of the Hom-space $\operatorname{Hom}_{\mathrm{K}^{b,-}(\operatorname{proj}(\Lambda))}\left(P_{M}^{\bullet}, \Sigma P_{N}^{\bullet}\right)$.

\section{Cohomology of STRING AND BAND COMPleXes}

Throughout $\sigma$ will be a (possibly infinite) homotopy string or band, unless one is specified explicitly. When we wish to specify that $\sigma$ is finite on the right we will write $\sigma=\cdots \sigma_{2} \sigma_{1}$, finite on the left: $\sigma=\sigma_{n} \sigma_{n-1} \cdots$, and finite on both sides: $\sigma=\sigma_{n} \cdots \sigma_{1}$.

Given a homotopy string or band $\sigma$ we will describe how to compute the cohomology of the string or band complex $Q_{\sigma}^{\bullet}$. The strategy is to divide $\sigma$ up into various homotopy substrings each corresponding to appropriately chosen two-term complexes. We start with an important technical definition.

Definition 2.1. Let $\sigma$ be a homotopy string or band. A homotopy substring $\tau=\sigma_{j} \cdots \sigma_{i}$ with $i<j$ is a maximal alternating homotopy substring if

(i) for each $i \leq k<j$, if $\sigma_{k}$ is direct (resp., inverse) then $\sigma_{k+1}$ is inverse (resp., direct);

(ii) if $\sigma_{i}$ is direct (resp., inverse) then $\sigma_{i-1}$ is direct (resp., inverse) and $\sigma_{i} \sigma_{i-1} \in I$ (resp., $\left.\overline{\sigma_{i-1} \sigma_{i}} \in I\right)$ or is $\varnothing$; and,

(iii) if $\sigma_{j}$ is direct (resp., inverse) then $\sigma_{j+1}$ is direct (resp., inverse) and $\sigma_{j} \sigma_{j+1} \in I$ (resp., $\overline{\sigma_{j+1} \sigma_{j}} \in I$ or is $\varnothing$.

If only condition (i) holds, then $\tau$ is called an alternating homotopy substring.

Remark 2.2. Let $\sigma$ be a homotopy string or band and $\tau=\sigma_{j} \cdots \sigma_{i}$ with $i<j$ be a maximal alternating homotopy substring of $\sigma$.

(1) The homotopy string $\tau$ has at least two homotopy letters.

(2) The string complex $P_{\tau}^{\bullet}$ is concentrated in precisely two cohomological degrees, namely $\operatorname{deg} P\left(s\left(\sigma_{i}\right)\right)$ and $\operatorname{deg} P\left(e\left(\sigma_{i}\right)\right)$, i.e. it is a 'two-term complex'.

(3) A maximal alternating homotopy substring of a homotopy string or band cannot be infinite: all infinite homotopy strings have antipaths to the left and/or to the right.

(4) Since no two consecutive homotopy letters of $\tau$ 'pass through a relation', the underlying walk of $\tau$ also determines a string. In the case that $\sigma=\sigma_{n} \cdots \sigma_{1}$ is a homotopy band and $\tau=\sigma$, then the underlying walk of $\tau$ also determines a band.

Lemma 2.3 (Maximal alternating homotopy substring rule). Let $\sigma$ be a homotopy string or band. Suppose $\tau=\sigma_{j} \cdots \sigma_{i}$ is a maximal alternating homotopy substring. Decompose the homotopy letters $\sigma_{j}=b_{l} \cdots b_{1}$ and $\sigma_{i}=a_{k} \cdots a_{1}$ into paths or inverse paths in $(Q, I)$ and set

$$
w:= \begin{cases}b_{l-1} \cdots b_{1} \sigma_{j-1} \cdots \sigma_{i+1} a_{k} \cdots a_{2} & \text { if } \tau \neq \sigma \text { or } \tau=\sigma \text { and } \sigma \text { is a homotopy string with } \sigma_{1} \\ \sigma & \text { if } \tau=\sigma \text { and } \sigma_{n} \text { direct }\end{cases}
$$

Then the string module $M(w)$ (resp., band module $B(w)$ ) is an indecomposable summand of the cohomology module $H^{d}\left(Q_{\sigma}^{\bullet}\right)$, where $d=\max \left\{\operatorname{deg} P\left(s\left(\sigma_{i}\right)\right), \operatorname{deg} P\left(e\left(\sigma_{i}\right)\right)\right\}$.

Proof. Suppose $\sigma$ is a homotopy string and $\left(Q_{\sigma}^{\bullet}, \partial^{\bullet}\right)$ is the corresponding string complex. We treat the case that the maximal alternating homotopy substring $\tau$ has unfolded diagram of the form below; the other cases, and the case that $\sigma$ is a homotopy band, are similar.

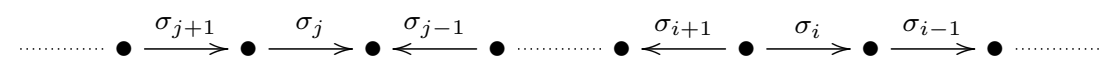


Note that, in this case $d=\operatorname{deg} P\left(s\left(\sigma_{i}\right)\right)$ and the homotopy letters $\sigma_{i}, \ldots, \sigma_{j}$ are components of the differential $\partial^{d-1}$. In particular, we can wrap $\tau$ back up into a complex:

$$
\begin{aligned}
& P\left(e\left(\sigma_{j+1}\right)\right) \stackrel{\sigma_{j+1}}{\longrightarrow} P\left(e\left(\sigma_{j}\right)\right) \stackrel{\sigma_{j}}{\longrightarrow} P\left(e\left(\sigma_{j-1}\right)\right)
\end{aligned}
$$

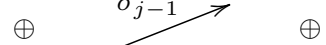

$$
\begin{aligned}
& P\left(e\left(\sigma_{j-2}\right)\right) \underset{\sigma_{j-3}}{\stackrel{\sigma_{j-2}}{\longrightarrow}} P\left(e\left(\sigma_{j-3}\right)\right) \\
& \oplus \frac{\sigma_{j-3}}{\sigma_{j-4}} \oplus \\
& P\left(e\left(\sigma_{j-4}\right)\right) \stackrel{\sigma_{j-4}}{\longrightarrow} \text { : } \\
& \oplus \quad \oplus \\
& \vdots \stackrel{\sigma_{i+2}}{\underset{\sigma_{i+1}}{\longrightarrow}} P\left(e\left(\sigma_{i+1}\right)\right) \\
& P\left(e\left(\sigma_{i}\right)\right) \stackrel{\oplus \sigma_{i}}{\longrightarrow} P\left(s\left(\sigma_{i}\right)\right) \stackrel{\oplus}{\longrightarrow} P\left(s\left(\sigma_{i-1}\right)\right)
\end{aligned}
$$

The other components of the differentials $\partial^{d-2}, \partial^{d-1}$ and $\partial^{d}$ are disconnected from the components of $\partial^{d-1}$ indicated above. The components above therefore contribute a summand, $M$ say, of the cohomology module $H^{d}\left(Q_{\sigma}^{\bullet}\right)$; the other summands of $H^{d}\left(Q_{\sigma}^{\bullet}\right)$ are contributed by other parts of $\sigma$. We claim that $M \cong M(w)$, where $w$ is the string defined in the statement.

The projective modules $P\left(e\left(\sigma_{i+1}\right)\right), P\left(e\left(\sigma_{i+3}\right)\right), \ldots, P\left(e\left(\sigma_{j-1}\right)\right) \subset \operatorname{ker}\left(\partial^{d}\right)$. Consider the following components of the differential $\partial^{d-1}$,

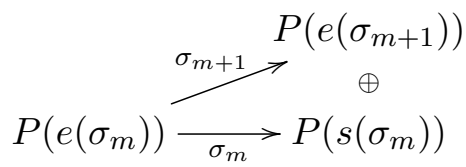

which map diagonally into submodules of $P\left(e\left(\sigma_{m+1}\right)\right)$ and $P\left(s\left(\sigma_{m}\right)\right)$ with simple top $S\left(e\left(\sigma_{m}\right)\right)$. Thus, in the quotient $\operatorname{ker}\left(\partial^{d}\right) / \operatorname{im}\left(\partial^{d-1}\right)$ the action of $\sigma_{m}$ on the basis vector at $s\left(\sigma_{m+1}\right)$ is the same as the action of $\sigma_{m-1}$ on the basis vector at $s\left(\sigma_{m-1}\right)$, as indicated in Figure 2. At the left-hand endpoint of $\tau$, i.e. at the homotopy letter $\sigma_{j}$, in the quotient $\operatorname{ker}\left(\partial^{d}\right) / \operatorname{im}\left(\partial^{d-1}\right)$ the arrow $b_{l}$ acts on the basis vector supported at $s\left(b_{l}\right)$ by sending it to 0 (because the basis vector at $e\left(b_{l}\right)$ is an element of $\operatorname{im}\left(\partial^{d-1}\right)$. Hence, the arrow $b_{l}$ is removed from the string describing this indecomposable summand of $H^{d}\left(P_{\sigma}^{\bullet}\right)$. Similarly, at the right-hand endpoint of $\tau$, i.e. at $\sigma_{i}$, the basis vector supported at $s\left(a_{1}\right)=x_{i-1}$ is not an element of $\operatorname{ker}\left(\partial^{d}\right)$. Hence, the arrow $a_{1}$ is removed from the string describing the indecomposable summand of $H^{d}\left(P_{\sigma}^{\bullet}\right)$. It follows that the summand $M$ of $\operatorname{ker}\left(\partial^{d}\right) / \operatorname{im}\left(\partial^{d-1}\right)$ has the following form.

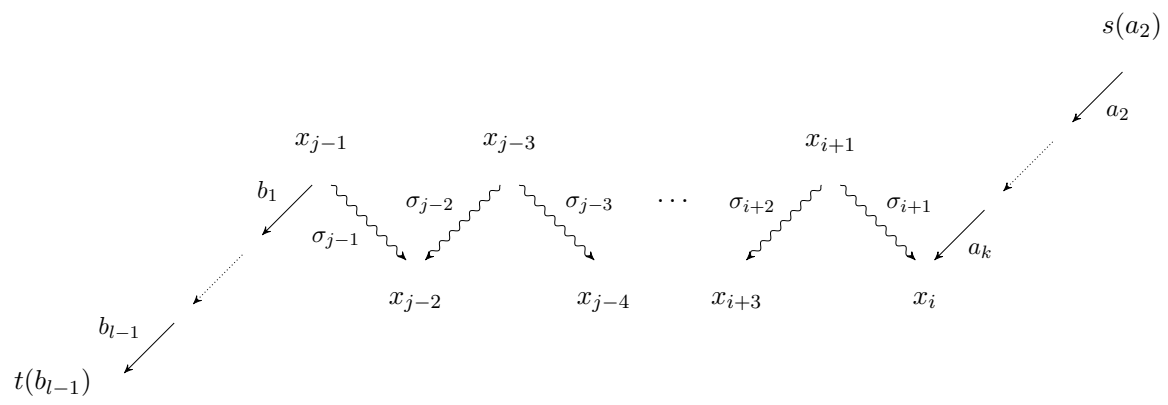

that is, corresponds to the string $w=b_{l-1} \cdots b_{1} \sigma_{j-1} \cdots \sigma_{i+1} a_{k} \cdots a_{2}$.

The following lemmas are computations analogous to that in Lemma 2.3 above. Thus we provide only their statements and leave the proofs to the reader.

Lemma 2.4 (Cokernel rule). Let $\sigma=\cdots \sigma_{2} \sigma_{1}$ be a homotopy string in which $\sigma_{1}=a_{k} \cdots a_{1}$ and $\sigma_{2}$ are direct homotopy letters. If there exists $c$ with $c \in \bar{Q}_{1}$ such that $\sigma_{1} c$ is defined as a string, 


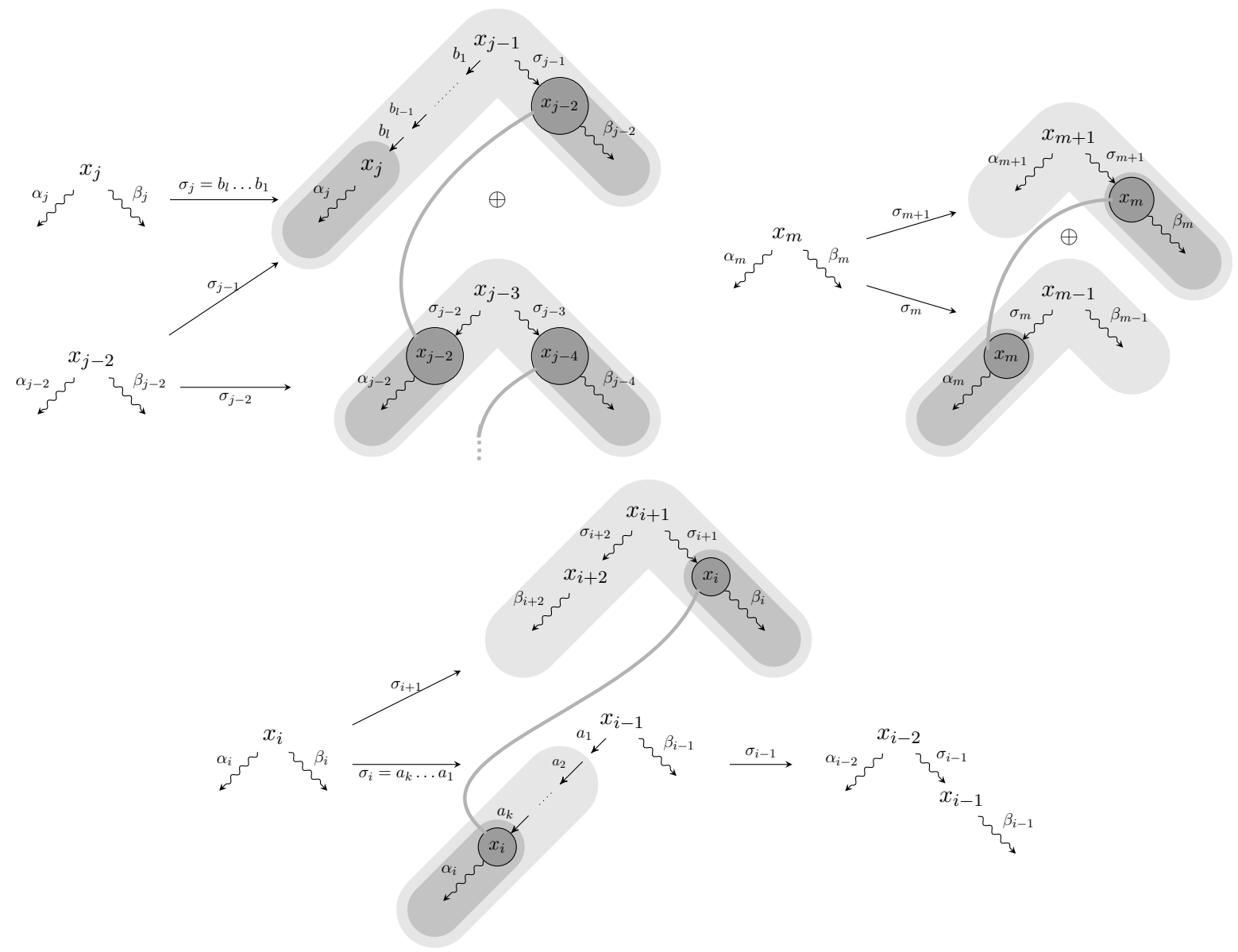

Figure 2. Schematic showing the computation of the cohomology: ker $\partial$ is shown in light grey, im $\partial$ in mid-grey, and basis vectors identified by quotienting by the image of the diagonal map shown in dark grey and joined by a dark grey line. Here $x_{m}=e\left(\sigma_{m}\right)$, i.e. is the end of the homotopy letter $\sigma_{m}$. Top left: illustration of the situation at the left end of the maximal alternating homotopy string $\tau$. Top right: a generic situation midway in $\tau$. Bottom: the situation at the right hand end of $\tau$.

then take $u=c_{m} \cdots c_{1}$ to be the maximal inverse string ending with $c_{m}=c$. Set

$$
w:= \begin{cases}a_{k-1} \cdots a_{1} u & \text { if there is such a } c \\ a_{k-1} \cdots a_{1} & \text { otherwise. }\end{cases}
$$

Then the string module $M(w)$ is an indecomposable summand of the cohomology module $H^{d}\left(P_{\sigma}^{\bullet}\right)$, where $d=\operatorname{deg} P\left(s\left(\sigma_{1}\right)\right)$.

For a homotopy string $\sigma=\cdots \sigma_{2} \sigma_{1}$ with $\sigma_{1}$ direct, it is possible that $\sigma_{2}$ is not direct, in which case $\sigma_{1}$ is part of a maximal alternating homotopy substring $\tau=\sigma_{j} \cdots \sigma_{1}$. In this case, we combine the cokernel rule with the maximal alternating homotopy substring rule.

Lemma 2.5 (Combined rule). Let $\sigma=\cdots \sigma_{2} \sigma_{1}$ be a homotopy string in which $\sigma_{1}=a_{k} \cdots a_{1}$ is a direct homotopy letter and $\tau=\sigma_{j} \cdots \sigma_{1}$ is a maximal alternating homotopy substring. Decompose the homotopy letter $\sigma_{j}=b_{l} \cdots b_{1}$ into a path or inverse path in $(Q, I)$ and set

$$
w:= \begin{cases}b_{l-1} \cdots b_{1} \sigma_{j-1} \cdots \sigma_{1} u & \text { if there exist } c \text { and } u \text { as in Lemma 2.4; } \\ b_{l-1} \cdots b_{1} \sigma_{j-1} \cdots \sigma_{2} a_{k} \cdots a_{1} & \text { otherwise. }\end{cases}
$$

Then the string module $M(w)$ is an indecomposable summand of the cohomology module $H^{d}\left(P_{\sigma}^{\bullet}\right)$, where $d=\operatorname{deg} P\left(s\left(\sigma_{1}\right)\right)$. 
There are obvious dual statements if $\sigma=\sigma_{n} \sigma_{n-1} \cdots$ with $\sigma_{n}$ inverse. If $\tau=\sigma$ with $\sigma_{n}$ inverse and $\sigma_{1}$ direct then we must combine Lemma 2.5 with its dual.

Lemma 2.6 (Kernel rule). Let $\sigma=\sigma_{n} \sigma_{n-1} \cdots$ be a homotopy string in which $\sigma_{n}=b_{l} \cdots b_{1}$ is a direct homotopy letter. If there exists $c \in Q_{1}$ and $c b_{l}=0$ then take $c_{m} \cdots c_{1}$ to be the maximal direct string starting with $c_{1}=c$. Set

$$
v:= \begin{cases}c_{m} \cdots c_{2} & \text { if there exists such a } c ; \\ \varnothing & \text { otherwise. }\end{cases}
$$

Then the string module $M(v)$ is an indecomposable summand of the cohomology module $H^{d}\left(P_{\sigma}^{\bullet}\right)$, where $d=\operatorname{deg} P\left(e\left(\sigma_{n}\right)\right)$. If $m=1$ then $v=1_{e(c)}$ is the trivial string corresponding to the simple module $S(e(c))$.

Note that if $\sigma=\sigma_{n} \sigma_{n-1} \cdots$ is a homotopy string and $\tau=\sigma_{n} \cdots \sigma_{i}$ is a maximal alternating homotopy substring with $\sigma_{n}$ direct, then Lemmas 2.3 and 2.6 do not need to be combined. In particular, the string module $M(v)$ is an indecomposable summand of $H^{d}\left(P_{\sigma}^{\bullet}\right)$ and the string module $M(w)$ is an indecomposable summand of $H^{d+1}\left(P_{\sigma}^{\bullet}\right)$, where $v$ is defined as in Lemma 2.6, $w$ is defined as in Lemma 2.3, and $d=\operatorname{deg} P\left(e\left(\sigma_{n}\right)\right)$.

Lemma 2.7 (Nontrivial homotopy letter rule). Let $\sigma$ be a homotopy string or band in which $\sigma_{i}$ is a direct homotopy letter and $\sigma_{i+1} \sigma_{i} \sigma_{i-1}$ is a non-alternating homotopy substring with $\sigma_{i+1}$ possibly empty. Let $d=\operatorname{deg} P\left(s\left(\sigma_{i}\right)\right)$.

(1) If $\sigma_{i}=a_{k} \cdots a_{1}$ with $a_{j} \in Q_{1}$ and $k>1$ then set $w=a_{k-1} \cdots a_{2}$. The string module $M(w)$ is an indecomposable summand of the cohomology module $H^{d}\left(Q_{\sigma}^{\bullet}\right)$. If $k=2$ then $w=1_{e\left(a_{1}\right)}=1_{s\left(a_{2}\right)}$ and $M(w)=S\left(e\left(a_{1}\right)\right)=S\left(s\left(a_{2}\right)\right)$.

(2) If $\sigma_{i}=a$ for some $a \in Q_{1}$ then the map $\sigma_{i}: P\left(e\left(\sigma_{i}\right)\right) \rightarrow P\left(s\left(\sigma_{i}\right)\right)$ contributes the zero submodule to the cohomology module of $H^{d}\left(Q_{\sigma}^{\bullet}\right)$.

Lemmas 2.4, 2.5, 2.6 and 2.7 admit obvious dual statements. When referring to these lemmas we shall freely include those dual statements. We summarise this section with the following theorem and illustrate with an example.

Theorem 2.8. Let $\sigma$ be a homotopy string or band. Lemmas 2.3, 2.4, 2.5, 2.6 and 2.7 and their duals provide a complete description of the cohomology complex $H^{\bullet}\left(Q_{\sigma}^{\bullet}\right)$.

Remark 2.9. Note that in computing the cohomology Lemmas 2.3, 2.4, 2.5, 2.6, 2.7 and their duals can be applied independently and therefore in any order. The only exception is that the combined rule Lemma 2.5 should always be applied instead of Lemma 2.3 whenever the homotopy string has the appropriate form.

Example 2.10. We consider the gentle algebra with the following quiver where the (length 2) relations are indicated by dotted lines.

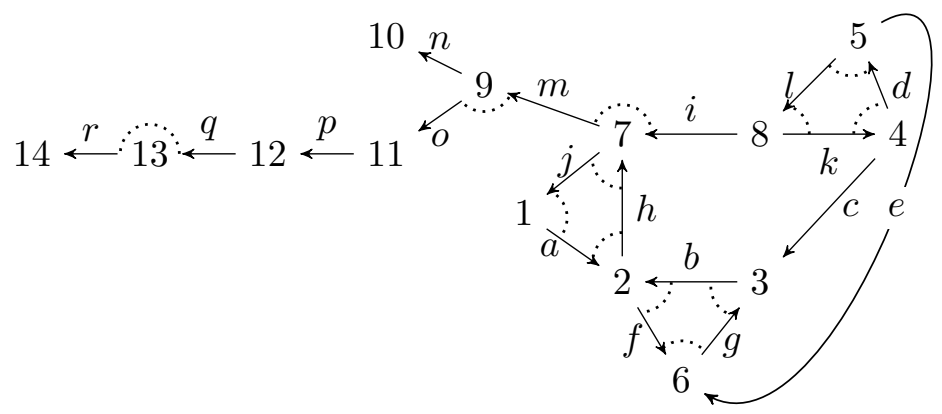


Consider the following homotopy strings where the top line indicates the cohomological degree of the corresponding projective indecomposable:

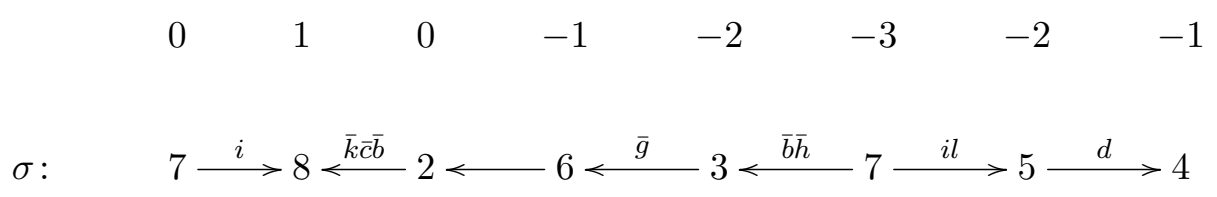

and

$$
\begin{array}{rrrrr} 
& -1 & 0 & 1 & 2 \\
\tau: & 14 \stackrel{r}{\longrightarrow} 13 \stackrel{q p o}{\longrightarrow} 9 \stackrel{m}{\longrightarrow} 7 .
\end{array}
$$

Examining the homotopy string $\sigma$, we see that there are four indecomposable summands of $H^{\bullet}\left(P_{\sigma}^{\bullet}\right)$. We list them below in order of ascending cohomological degree.

- We have $H^{-2}\left(P_{\sigma}^{\bullet}\right)=M\left(w_{1}\right)$, where $w_{1}=\bar{h} i$ coming from the maximal alternating homotopy substring rule (Lemma 2.3) applied to $3 \stackrel{\bar{b} \bar{h}}{\longleftarrow} 7 \stackrel{i l}{\longrightarrow} 5$.

- We have $H^{-1}\left(P_{\sigma}^{\bullet}\right)=M\left(w_{2}\right)$, where $w_{2}=\bar{c} \bar{b} \bar{h} \bar{m} \bar{n}$ coming from the cokernel rule (Lemma 2.4) applied to $5 \stackrel{d}{\longrightarrow} 4$.

- We have $H^{0}\left(P_{\sigma}^{\bullet}\right)=M\left(w_{3}\right)$, where $w_{3}=n$ coming from the kernel rule (Lemma 2.6) applied to $7 \stackrel{i}{\longrightarrow} 8$.

- We have $H^{1}\left(P_{\sigma}^{\bullet}\right)=M\left(w_{4}\right)$, where $w_{4}=\bar{k} \bar{c}$ coming from the maximal alternating homotopy substring rule (Lemma 2.3) applied to $7 \stackrel{i}{\longrightarrow} 8 \stackrel{\bar{k} \bar{c} \bar{b}}{\longleftarrow} 2$.

- By Lemma 2.7, all remaining parts of the homotopy string $\sigma$ contribute zero to the cohomology $H^{\bullet}\left(P_{\sigma}^{\bullet}\right)$.

Examining the homotopy string $\tau$, in a similar fashion we obtain the following for $H^{\bullet}\left(P_{\tau}^{\bullet}\right)$.

- We have $H^{1}\left(P_{\tau}\right)=M\left(w_{1}\right)$ where $w_{1}=p$ coming from the nontrivial homotopy letter rule (Lemma 2.7) applied to $13 \stackrel{q p o}{\longrightarrow} 9 \stackrel{m}{\longrightarrow} 7$.

- We have $H^{2}\left(P_{\tau}\right)=M\left(w_{2}\right)$ where $w_{2}=j$ coming from the cokernel rule (Lemma 2.4) applied to $9 \stackrel{m}{\longrightarrow} 7$.

- There is no non-zero contribution to the cohomology coming from the nontrivial homotopy letter rule (Lemma 2.7) applied to $14 \stackrel{r}{\longrightarrow} 13 \stackrel{q p o}{\longrightarrow} 9$ or from the kernel rule (Lemma 2.6) applied to $14 \stackrel{r}{\longrightarrow} 13$.

We end this section by giving the homotopy string or band of the minimal projective resolution of a string or quasi-simple band module, which will be heavily used in the next sections. We note that gentle algebras are string algebras and that there is a large body of work on string algebras. In particular, projective resolutions and syzygies, have been considered before, see for example [17, 18]. In [28], minimal projective presentations of string and band modules were given in terms of string combinatorics, which in the case of gentle algebras can be formulated in terms of homotopy string combinatorics. These projective presentations correspond to maximal alternating homotopy substrings sitting between degrees -1 and 0 . Before stating the result, we set up some notation.

Definition 2.11. Let $a$ and $b$ be such that $\bar{a}, b \in Q_{1}$. Define

- $\operatorname{inv}(a):=\sigma_{-1} \sigma_{-2} \cdots$ to be maximal inverse antipath ending with $\sigma_{-1}=a$;

- $\operatorname{dir}(b):=\cdots \sigma_{2} \sigma_{1}$ to be the maximal direct antipath starting with $\sigma_{1}=b$.

Corollary 2.12. Let $w=w_{n} \cdots w_{1}$ be a string. Define a homotopy string $\pi(w)$ as follows:

(1) $\pi(w)=\operatorname{dir}(b) w^{\prime} \operatorname{inv}(a)$ if there are $a$ and $b$ such that $\bar{a}, b \in Q_{1}$ and bwa is defined as $a$ string and where $w^{\prime}=w$. 
(2) $\pi(w)=w^{\prime} \operatorname{inv}(a)$ if there is an a with $\bar{a} \in Q_{1}$ such that wa is defined as a string but $n o b \in Q_{1}$ with $b w$ defined as a string, where $w^{\prime}=w_{j} \cdots w_{1}$ after removing a maximal direct substring $w_{n} \cdots w_{j+1}$ of $w$.

(3) $\pi(w)=\operatorname{dir}(b) w^{\prime}$ if there is $b \in Q_{1}$ with bw defined as a string but no a with $\bar{a} \in Q_{1}$ such that wa is defined as a string, where $w^{\prime}=w_{n} \cdots w_{i}$ after removing a maximal inverse substring $w_{i-1} \cdots w_{1}$ of $w$.

(4) $\pi(w)=w^{\prime}$ if there are no $a$ and $b$ such that $\bar{a}, b \in Q_{1}$ and bwa is defined as a string, where $w^{\prime}=w_{j} \cdots w_{i}$ after removing a maximal direct substring $w_{n} \cdots w_{j+1}$ and a maximal inverse substring $w_{i-1} \cdots w_{1}$.

(5) $\pi(w)=w$ if $w$ is a band.

Then $P_{\pi(w)}^{\bullet}$ (resp., $B_{\pi(w)}^{\bullet}$ when $w$ is a band) is a projective resolution of $M(w)$ (resp., $B(w)$ ).

Proof. The computation of the cohomology of $P_{\pi(w)}^{\bullet}$ (resp., $\left.B_{\pi(w)}^{\bullet}\right)$ in Theorem 2.8 gives $M(w)$ (resp., $B(w)$ ) in cohomological degree zero and zero in all other degrees.

Corollary 2.13. Let $A$ be a gentle algebra. Then any quasi-simple band module has projective dimension one.

The maximal direct substring $w_{n} \cdots w_{j+1}$ removed from $w$ in Corollary 2.12(2) will be called a maximal direct suffix. Likewise, the maximal inverse substring $w_{i-1} \cdots w_{1}$ removed from $w$ in Corollary 2.12(3) will be called a maximal inverse prefix.

Definition 2.14. For the homotopy string $\sigma=\pi(w)$ defined in Corollary 2.12 above we call the homotopy substrings $\operatorname{inv}(a)$ and $\operatorname{dir}(b)$ the antipath part of $\pi(w)$. By abuse of notation we write $\operatorname{inv}(w)=\operatorname{inv}(a)$ and $\operatorname{dir}(w)=\operatorname{dir}(b)$. In the notation of Corollary 2.12, we will call $w^{\prime}$ the module part of $\pi(w)$.

An inverse homotopy letter $\sigma_{i}=\bar{a}_{1} \cdots \bar{a}_{k}$ of $\sigma$ is incident with $\operatorname{inv}(a)$ if $\bar{a}_{k}=\bar{a}$. Likewise, a direct homotopy letter $\sigma_{j}=b_{l} \cdots b_{1}$ of $\sigma$ is incident with $\operatorname{dir}(b)$ if $b_{1}=b$.

In the following, as usual, we write $Q_{\pi(w)}^{\bullet}$ when we do not wish to specify whether $w$ is a string or a band.

Remark 2.15. We make the following straightforward observations regarding the forms of the homotopy strings occurring in Corollary 2.12.

(1) If there is no $a$ such that $w a$ is defined as a string then the homotopy string $\pi(w)$ starts with a direct homotopy letter whose target lies in degree 0 .

(2) If there is no $b$ such that $b w$ is defined as a string then the homotopy string $\pi(w)$ ends with an inverse homotopy letter whose target lies in degree 0 .

(3) If $\sigma_{i+1} \sigma_{i}$ are consecutive homotopy letters with the same orientation then at least one of them lies in the antipath part and the other either lies in the antipath part or else is incident with $\operatorname{dir}(w)$ or $\operatorname{inv}(w)$.

(4) Owing to being a projective resolution of a module, the string/band complex $Q_{\pi(w)}^{\bullet}$ attains its maximal cohomological degree in degree 0 . Moreover, homotopy letters occurring in the module part of $\pi(w)$ provide components of the differential in $Q_{\pi(w)}^{\bullet}$ from degree -1 to degree 0 . Indeed, together with those homotopy letters incident with $\operatorname{dir}(w)$ and $\operatorname{inv}(w)$ these provide all components of the differential in $Q_{\pi(w)}^{\bullet}$ from degree -1 to degree 0 .

(5) Suppose $\sigma_{k}$ is a homotopy letter of $\pi(w)$. If length $\left(\sigma_{k}\right)>1$ then $\operatorname{deg}\left(P\left(e\left(\sigma_{k}\right)\right)\right) \in\{0,-1\}$ and $\operatorname{deg}\left(P\left(s\left(\sigma_{k}\right)\right)\right) \in\{-1,0\}$, where $\operatorname{deg}(P(x))$ denotes the cohomological degree in which $P(x)$ occurs.

\section{Determining extensions in the module Category}

Recall that in [25] extensions for string modules are given in terms of string combinatorics. 
Definition 3.1. Let $v$ be a string or band and $w$ be a string or band.

(1) (Arrow extension) If there exists $a \in Q_{1}$ such that $u=w a v$ is a string then there is a non-split short exact sequence

$$
0 \rightarrow M(w) \rightarrow M(u) \rightarrow M(v) \rightarrow 0 .
$$

(2) (Overlap extension) Suppose that $v=v_{L} \bar{B} m A v_{R}$ and $w=w_{L} D m \bar{C} w_{R}$ with $A, B, C, D \in Q_{1}$ and $m, v_{L}, v_{R}, w_{L}, w_{R}$ (possibly trivial) strings such that

(i) if $A=\varnothing$ then $C \neq \varnothing$;

(ii) if $B=\varnothing$ then $D \neq \varnothing$; and,

(iii) if $m=1_{x}$ for some $x \in Q_{0}$, i.e. a trivial string, then $C A \in I$ and $B D \in I$ (whenever they exist, subject to the constraints above).

Then there is a non-split short exact sequence

$$
0 \rightarrow M(w) \rightarrow M(u) \oplus M\left(u^{\prime}\right) \rightarrow M(v) \rightarrow 0
$$

where $u=w_{L} D m A v_{R}$ and $u^{\prime}=v_{L} \bar{B} m \bar{C} w_{R}$.

Remark 3.2. Condition (iii) is a 'compatibly oriented' condition corresponding to [9, Def 2.1]. We remark that this condition is missing in the definition of overlap extension in [11], but it is used implicitly in the arguments therein.

Recall the canonical isomorphism (1) from the introduction,

$$
\Phi: \operatorname{Hom}_{\mathrm{K}^{b,-}(\operatorname{proj}(\Lambda))}\left(Q_{\pi(v)}^{\bullet}, \Sigma Q_{\pi(w)}^{\bullet}\right) \stackrel{\sim}{\rightarrow} \operatorname{Ext}_{\Lambda}^{1}(M(v), M(w)) .
$$

Theorem 3.3. With the notation above, let $M(v)$ and $M(w)$ be indecomposable $\Lambda$-modules with strings or bands $w$ and $v$ respectively and let $Q_{\sigma}^{\bullet}$ and $Q_{\tau}^{\bullet}$ with $\sigma=\pi(v)$ and $\tau=\pi(w)$ be their projective resolutions. Then for any standard basis element $f^{\bullet}$ in $\operatorname{Hom}_{\mathrm{K}^{b,-}(\operatorname{proj}(\Lambda))}\left(Q_{\sigma}^{\bullet}, \Sigma Q_{\tau}^{\bullet}\right)$ the corresponding extension $\Phi\left(f^{\bullet}\right)$ in $\operatorname{Ext}_{\Lambda}^{1}(M(v), M(w))$ is given by an arrow or an overlap extension. In particular, the set of overlap and arrow extensions form a generating set for $\operatorname{Ext}_{\Lambda}^{1}(M(v), M(w))$.

In the rest of this section we prove Theorem 3.3 by considering each type of map of the standard basis of $\operatorname{Hom}_{\mathrm{K}^{b,-}(\operatorname{proj}(\Lambda))}\left(Q_{\sigma}^{\bullet}, \Sigma Q_{\tau}^{\bullet}\right)$ as defined in [1]. We start by showing that Theorem 3.3 holds for graph maps.

3.1. Graph maps. Throughout this subsection we fix the following setup.

Setup 3.4. Let $v$ and $w$ be strings or bands and $M(v)$ and $M(w)$ be the corresponding string or band modules. Let $\sigma=\pi(v)$ and $\tau=\pi(w)$ be the homotopy strings or bands corresponding to the projective resolutions $Q_{\sigma}^{\bullet}$ and $Q_{\tau}^{\bullet}$ of $M(v)$ and $M(w)$ as given in Corollary 2.12, respectively.

Lemma 3.5. Let $f^{\bullet}: Q_{\sigma}^{\bullet} \rightarrow \Sigma Q_{\tau}^{\bullet}$ be a graph map incident with an antipath in $Q_{\sigma}^{\bullet}$ and an antipath in $\Sigma Q_{\tau}^{\bullet}$. Then $\Phi\left(f^{\bullet}\right)$ is an arrow extension in $\operatorname{Ext}_{\Lambda}^{1}(M(v), M(w))$.

Proof. Recall that $\pi(v)=\sigma$ and $\pi(w)=\tau$. We treat the case $\pi(v)=\operatorname{dir}(a) v \varphi$ and $\pi(w)=$ $\varphi^{\prime} w \operatorname{inv}\left(a^{\prime}\right)$ in detail, where $a, \bar{a}^{\prime} \in Q_{1}, \varphi$ is an inverse antipath and $\varphi^{\prime}$ is a direct antipath, putting $\pi(v)$ and $\pi(w)$ both in case (1) of Corollary 2.12. If one of $\varphi=\varnothing$ or $\varphi^{\prime}=\varnothing$, i.e. if $\pi(v)=\operatorname{dir}(a) v^{\prime}$ or $\pi(w)=w^{\prime} \operatorname{inv}\left(a^{\prime}\right)$, then the calculations below remain essentially the same, even in the case that $v$ is inverse and $w$ is direct.

To simplify the notation, set $\operatorname{dir}(a)=\theta=\cdots \theta_{2} \theta_{1}$ and $\operatorname{inv}\left(a^{\prime}\right)=\theta^{\prime}=\theta_{1}^{\prime} \theta_{2}^{\prime} \cdots$ with $\theta_{i}, \bar{\theta}_{i}^{\prime} \in Q_{1}$ and $\theta_{1}=a$ and $\theta_{1}^{\prime}=a^{\prime}$. Suppose that $f^{\bullet}$ induces an isomorphism of projective modules lying in $\theta$ and $\theta^{\prime}$ and suppose this isomorphism is in degree $-n$. Then as homotopy letters in antipaths are of length 1 and since $\Lambda$ is gentle, there exists an isomorphism $\theta_{n} \simeq \bar{\theta}_{n-1}^{\prime}$ and we obtain an isomorphism in degree $-n-1$. We now continue inductively to the left and right; we only need to take care about what happens in degree -1 , which we analyse in cases below. Write $v=v_{k} \cdots v_{1}$ and $w=w_{l} \cdots w_{1}$.

Case 1: $v_{k}$ is inverse and $w_{1}$ is direct. 
We have the following unfolded diagram

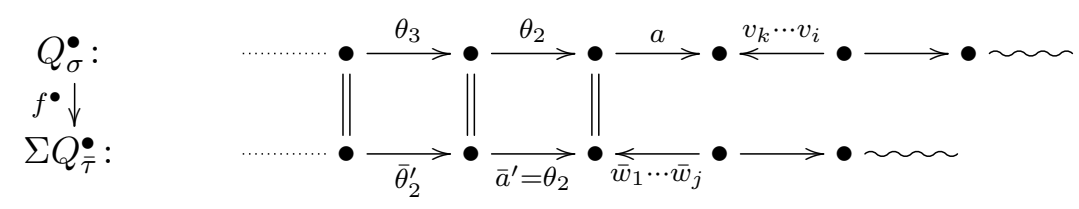

where $1 \leq i \leq k$ and $1 \leq j \leq l$. Then by [9, Thm. 2.2] the homotopy string of the (shift of the) mapping cone of $f^{\bullet}$ is given by $\alpha=\varphi^{\prime}$ wav $\varphi$. By the form of $\pi(w)$ and $\pi(v)$, it follows from Corollary 2.12 that there exist $\bar{b}, b^{\prime} \in Q_{1}$ such that $\varphi=b \rho$ and $\varphi^{\prime}=\rho^{\prime} b^{\prime}$ and $b^{\prime} w a v b$ is a string. Then by Lemma 2.3, M(wav) is the cohomology (in cohomological degree zero) of $Q_{\alpha}^{\bullet}$.

The shift of the mapping cone, $Q_{\alpha}^{\bullet}$, by definition sits in a distinguished triangle

$$
Q_{\tau}^{\bullet} \stackrel{h^{\bullet}}{\longrightarrow} Q_{\alpha}^{\bullet} \stackrel{g^{\bullet}}{\longrightarrow} Q_{\sigma}^{\bullet} \stackrel{f^{\bullet}}{\longrightarrow} \Sigma Q_{\tau}^{\bullet}
$$

We now observe that $H^{0}\left(g^{\bullet}\right):=g: M(w a v) \rightarrow M(v)$ is the canonical map in the arrow extension, showing that the corresponding graph map does indeed induce the claimed arrow extension.

Decompose $v=\nu_{n} \cdots \nu_{1}$ and $w=\mu_{m} \cdots \mu_{1}$ into homotopy letters so that $\sigma=\theta \nu_{n} \cdots \nu_{1} b \rho$ and $\alpha=\varphi^{\prime} \mu_{m} \cdots \mu_{1} a \nu_{n} \cdots \nu_{1} b \rho$. We assume that $\nu_{1}$ is direct so that $b$ is a homotopy letter; the case $\nu_{1}$ is inverse is similar. The map $g^{\bullet}: Q_{\alpha}^{\bullet} \rightarrow Q_{\sigma}^{\bullet}$ is given by the following unfolded diagram

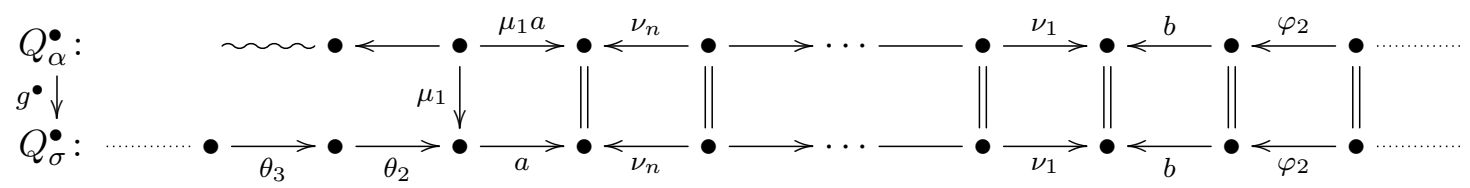

which is supported in cohomological degree -1 at the left endpoint. Wrapping $\alpha$ and $\sigma$ back up into complexes as in the proof of Lemma 2.3, where we have taken a 'mirror image' of $\sigma$ in order to more easily match up the cohomological degree 0 parts, we get the following diagram.

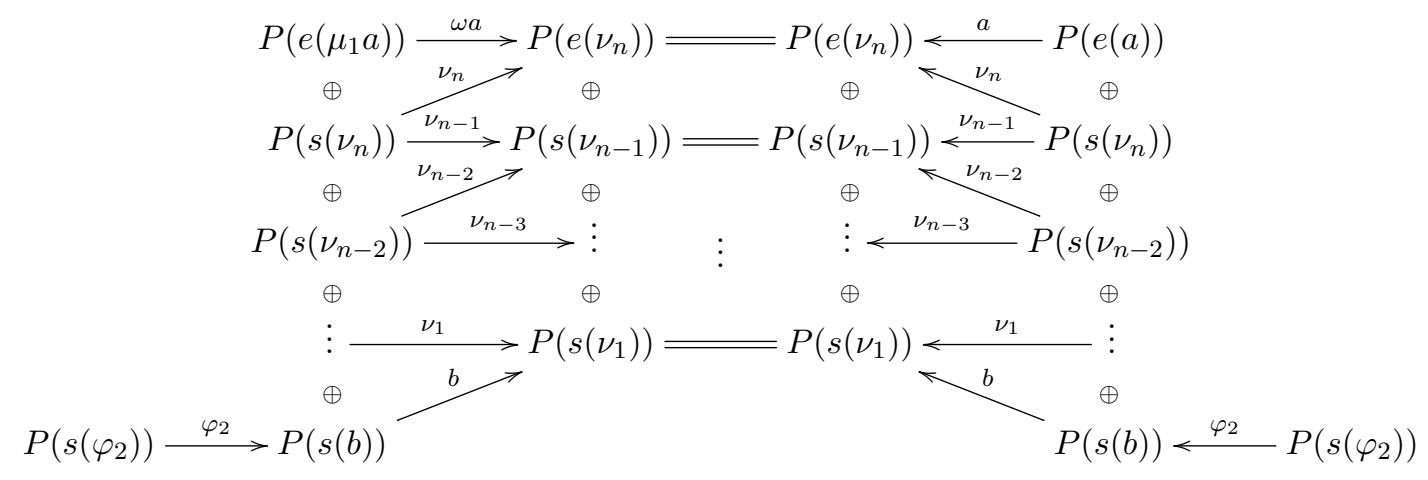

In Figure 3, we re-write diagram (7) as in Figure 2. Here we see immediately that $H^{0}\left(g^{\bullet}\right)$ is the canonical factor map $M(w a v) \rightarrow M(v)$. Taking the long exact cohomology sequence associated to the triangle (6) gives a short exact sequence

$$
0 \longrightarrow M(w) \stackrel{H^{0}\left(h^{\bullet}\right)}{\longrightarrow} M(w a v) \stackrel{H^{0}\left(g^{\bullet}\right)}{\longrightarrow} M(v) \longrightarrow 0,
$$

in which $H^{0}\left(g^{\bullet}\right)$ is the canonical map, whence it follows immediately that $H^{0}\left(h^{\bullet}\right)$ is also the canonical map associated to the obvious substring/factor string decomposition.

It now follows that $f^{\bullet}$ induces an arrow extension corresponding to the arrow induced by $a$, where the middle term of the extension is given by the string module $M(w a v)$.

Case 2: Both $v_{k}$ and $w_{1}$ are inverse. 


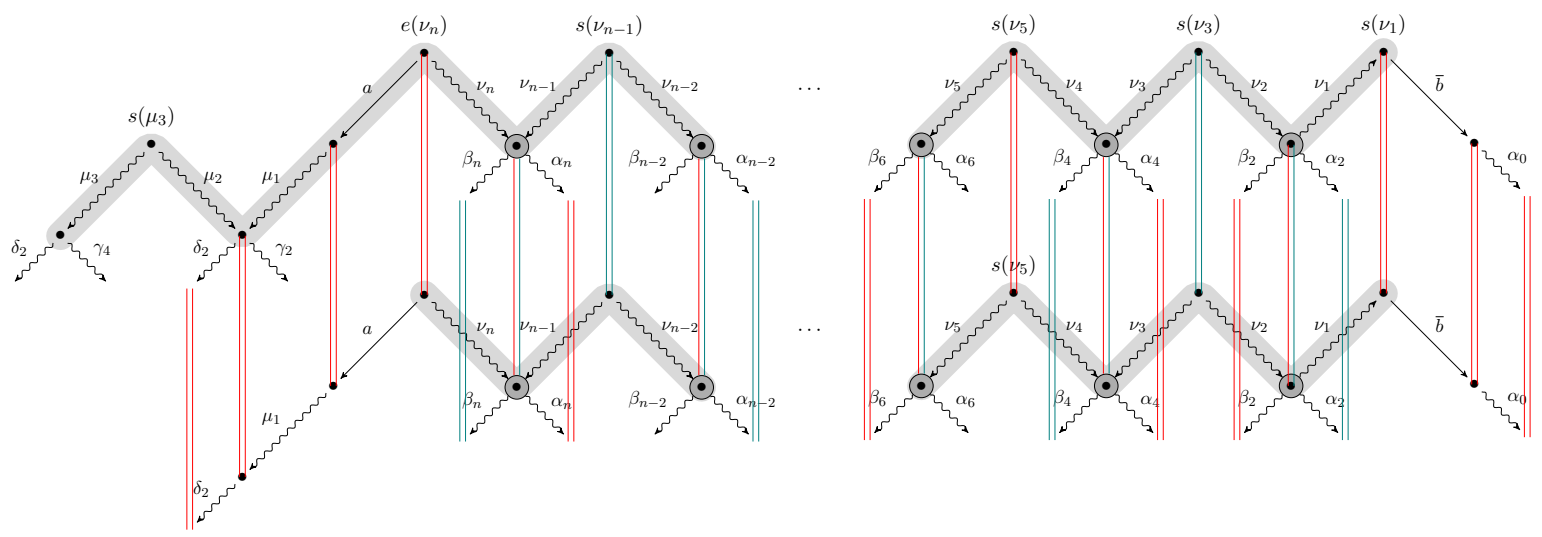

Figure 3 . The strings shaded in grey are the cohomology of $Q_{\alpha}^{\bullet}$ and $Q_{\sigma}^{\bullet}$ in degree 0 . The identity maps between projective modules are indicated in alternating red and green colour. The induced map between cohomology modules is the canonical factor map $M(w a v) \rightarrow M(v)$.

We have the following unfolded diagram


where $1 \leq i \leq k$ and $1 \leq j \leq l$. Then $a^{\prime}=\theta_{1}$ and by [9, Thm. 2.2] the homotopy string of the mapping cone of $f^{\bullet}$ is given by $\alpha=\rho^{\prime} b^{\prime}$ wavb $\rho$ where $\varphi=b \rho$ and $\varphi^{\prime}=\rho^{\prime} b^{\prime}$ with $\bar{b}, b^{\prime} \in Q_{1}$ such that $b^{\prime} w a v b$ is a string. As in Case 1 above, one can check that the map $H^{0}\left(g^{\bullet}\right): M($ wav $) \rightarrow$ $M(v)$ is the canonical map given by the obvious substring/factor string decomposition. It then follows that, taking cohomology, $f^{\bullet}$ induces an arrow extension, corresponding to the arrow $a$, whose middle term is $M($ wav $)$.

Case 3: Both $v_{k}$ and $w_{1}$ are direct.

This case is similar to case 1 . We have the following unfolded diagram

$$
\begin{gathered}
Q_{\sigma}^{\bullet}: \\
f^{\bullet} \downarrow \\
\Sigma Q_{\bar{\tau}}^{\bullet}:
\end{gathered}
$$

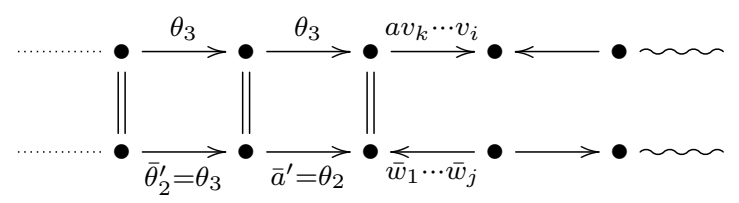

where $1 \leq i \leq k$ and $1 \leq j \leq l$. Then as above the cohomology of the mapping cone induces an arrow extension corresponding to the arrow $a$.

Case 4: $v_{k}$ is direct and $w_{1}$ is inverse.

This case is similar to case 2 . We have the following unfolded diagram

$$
\begin{gathered}
Q_{\sigma}^{\bullet}: \\
f^{\bullet} \downarrow \\
\Sigma Q_{\overline{\bar{\tau}}}^{\bullet}:
\end{gathered}
$$



where $1 \leq i \leq k$ and $1 \leq j \leq l$. Then as above the cohomology of the mapping cone induces an arrow extension corresponding to the arrow $a$.

Case 5: $v$ or $w$ or both are trivial. 
If $v$ is trivial but $w$ is not, this is a degenerate case of Case 1 or 2 . If $v$ is not trivial but $w$ is, this is a degenerate case of Case 1 or 3 . If both $v$ and $w$ are trivial, this is a degenerate case of Case 1.

Lemma 3.6. Let $f^{\bullet}: Q_{\sigma}^{\bullet} \rightarrow \Sigma Q_{\tau}^{\bullet}$ be a graph map and let $\nu$ in $\sigma$ and $\omega$ in $\tau$ be the maximal alternating homotopy substrings corresponding to the module parts of $\sigma$ and $\tau$ respectively. Suppose that $f^{\bullet}$ is supported in projective modules lying in $\nu$ and $\omega$. Then $f^{\bullet}$ is supported in a single indecomposable projective $\Lambda$-module $P$ in degree -1 unless it is incident with antipaths in both $Q_{\sigma}^{\bullet}$ and $\Sigma Q_{\tau}^{\bullet}$.

Furthermore, $\Phi\left(f^{\bullet}\right)$ gives rise to either an arrow extension or an overlap extension where the overlap is given by the simple $\Lambda$-module $P / \operatorname{rad}(P)$.

Proof. There are three cases to be considered.

Case 1: $f^{\bullet}$ is supported in $\nu$, and $f^{\bullet}$ is not incident with any antipath of $\sigma$.

In this case, we must have at least one isomorphism between projective modules in degree -1 as follows

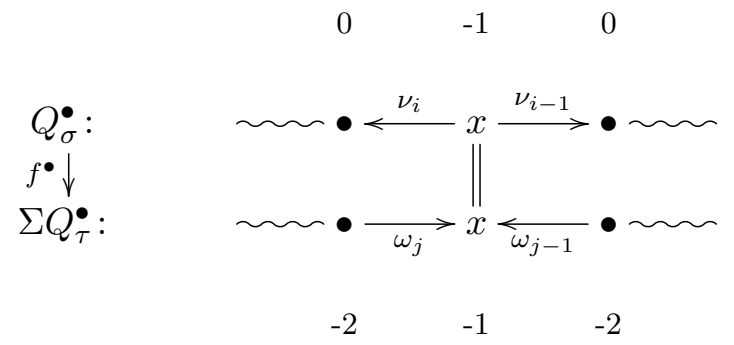

where $x \in Q_{0}$. Since the projectives in $\nu$ as a substring of $\sigma$ are in cohomological degrees 0 and -1 and the projectives in $\omega$ as a substring of the homotopy string corresponding to $\Sigma Q_{\tau}^{\bullet}$ are in degrees -1 and -2 , the graph map $f^{\bullet}$ can only be supported in a single degree, as shown. By reversing the orientation on $\tau$ if necessary, we may assume that $\sigma$ and $\tau$ are compatibly oriented in the sense of [9, Def 2.1].

Now, the homotopy letters $\nu_{i-1}, \nu_{i}, \omega_{j-1}$ and $\omega_{j}$ have the form $\nu_{i-1}=A \nu_{i-1}^{\prime}, \nu_{i}=\nu_{i}^{\prime} \bar{B}$, $\omega_{j-1}=\bar{D} \omega_{j-1}^{\prime}$ and $\omega_{j}=\omega_{j}^{\prime} C$, where $A, B, C, D \in Q_{1}$ and the primed symbols are homotopy subletters. Then $v=v_{L} \bar{B} A v_{R}$ and $w=w_{L} D \bar{C} w_{R}$ where $v_{L}, v_{R}$ are (possibly trivial) subwords of $v$ and $w_{L}, w_{R}$ are (possibly trivial) subwords of $w$. Set $x=e(A)(=e(\bar{B})=s(\bar{C})=s(D))$. We wish to verify we have an overlap extension in which $m=1_{x}$ in the sense of Definition 3.1(2).

First observe that whenever $A, B, C, D$ exist, the fact that diagram (8) is compatibly oriented means that $C A \in I$ and $B D \in I$. We now need to check that if $A=\varnothing$ then $C \neq \varnothing$ and if $B=\varnothing$ then $D \neq \varnothing$. We check the first condition, the second is analogous.

Suppose that $C=\varnothing$, i.e. $w=w_{L} D$. If $A=\varnothing$ then $v=v_{L} \bar{B}$. There must be an arrow $a \in Q_{1}$ such that $v \bar{a}$ is defined as a string, otherwise by Corollary 2.12, $\bar{B}$ would be removed as a maximal inverse prefix and the situation depicted in the unfolded diagram (8) would not occur. However, in this case the homotopy letters $\nu_{i}=\nu_{i}^{\prime} \bar{B} \bar{a}$ and $\nu_{i-1}$ must be inverse or empty, again taking us outside the situation occurring in diagram (8). Hence, we must have $A \neq \varnothing$, as required. A similar argument shows that if $B=\varnothing$ then $D \neq \varnothing$. We have thus verified that the conditions for an overlap extension in Definition 3.1(2) hold.

Finally, by $[9, \S 2]$ the mapping cone of $f^{\bullet}$ is a direct sum of the projective resolutions of the $\Lambda$-modules $M(u)$ and $M\left(u^{\prime}\right)$ where $u=w_{L} D e_{x} A v_{R}$ and $u^{\prime}=v_{L} \bar{B} e_{x} \bar{C} w_{R}$. Taking cohomology and checking the maps in the corresponding triangle as in the proof of Lemma 3.5 then shows that $f^{\bullet}$ gives rise to an overlap extension in the simple $\Lambda$-module $S(x)$.

Case 2: $f^{\bullet}$ is incident with an antipath in $Q_{\sigma}^{\bullet}$ and the module part in $\Sigma Q_{\tau}^{\bullet}$. 
In this case we obtain the following diagram for $f^{\bullet}$.

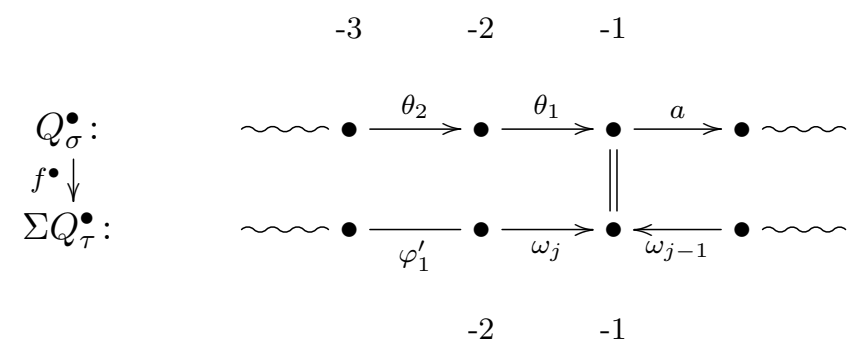

Since $\theta_{1}$ is a homotopy letter of length 1 , in order to obtain a graph map supported in more than one degree, we must have $\theta_{1}=\omega_{j}$. If $\varphi_{1}^{\prime}$ is inverse or empty then we reach a non-commuting endpoint condition as defined in Section 1.4.4, see also $[9, \S 1.4 .4]$ for more details on noncommuting endpoint conditions. This contradicts the fact that $f^{\bullet}$ is a graph map. Thus $\varphi_{1}^{\prime}$ must be direct and $\varphi_{1}^{\prime}=\theta_{2}$. We are therefore in the setup of Lemma 3.5 and the corresponding extension in the module category is an arrow extension.

Case 3: $f^{\bullet}$ is incident with the module part in $Q_{\sigma}^{\bullet}$ and an antipath in $\Sigma Q_{\tau}^{\bullet}$

This case cannot happen for degree reasons.

Remark 3.7. Suppose one of $v$ or $w$ is a band, in which case $\sigma$ or $\tau$ is a homotopy band. A priori, one might expect that there may be a graph map $f^{\bullet}: Q_{\sigma}^{\bullet} \rightarrow \Sigma Q_{\tau}^{\bullet}$ determined by an overlap which is longer than $\sigma$ or $\tau$. However, Lemmas 3.5 and 3.6 ensure that this situation can never occur. This makes sense: in $[10, \S 1]$ such a situation corresponds to a 'shortening' of the homotopy string or homotopy band determining the mapping cone relative to those occurring in the domain and target of the map. This would correspond to having a middle term of an extension of smaller dimension than the sum of the outer terms.

3.2. Quasi-graph maps. In this section we consider a quasi-graph map $\varphi: Q_{\sigma}^{\bullet} \rightsquigarrow$ $Q_{\tau}^{\bullet}$, corresponding to a homotopic family of single and double maps in the basis of $\operatorname{Hom}_{\mathrm{K}^{b,-}(\operatorname{proj}(\Lambda))}\left(Q_{\sigma}^{\bullet}, \Sigma Q_{\tau}^{\bullet}\right)$; see [1, Def. 3.12].

We start by placing a restriction on the cohomological degrees in which a quasi-graph map $\varphi: Q_{\sigma}^{\bullet} \rightsquigarrow Q_{\tau}^{\bullet}$ can be supported.

Lemma 3.8. Under the hypotheses of Setup 3.4, a quasi-graph map $\varphi: Q_{\sigma}^{\bullet} \rightsquigarrow Q_{\tau}^{\bullet}$ is supported in cohomological degrees -1 and 0 only.

Proof. If one of $Q_{\sigma}^{\bullet}$ or $Q_{\tau}^{\bullet}$ is a band complex then, by Corollary 2.13, it is supported in cohomological degrees -1 and 0 only, and therefore any quasi-graph map $\varphi: Q_{\sigma}^{\bullet} \rightsquigarrow Q_{\tau}^{\bullet}$ is trivially supported in only those cohomological degrees. Therefore we assume that $Q_{\sigma}^{\bullet}=P_{\sigma}^{\bullet}$ and $Q_{\tau}^{\bullet}=P_{\tau}^{\bullet}$ are string complexes.

Suppose, for a contradiction, that $\varphi: P_{\sigma}^{\bullet} \rightsquigarrow P_{\tau}^{\bullet}$ is supported in cohomological degree $-k \leq-2$. By Remark 2.15(4), any component of $\varphi$ supported in degrees $-k \leq-2$ occurs in antipath parts of $P_{\sigma}^{\bullet}$ and $P_{\tau}^{\bullet}$. Without loss of generality, we may assume, up to inversion if necessary, that $\sigma=\operatorname{dir}(b) w^{\prime} \sigma_{R}$ and $\tau=\operatorname{dir}(d) v^{\prime} \tau_{R}$, where $\sigma_{R}$ is either an inverse antipath or empty; likewise for $\tau_{R}$. Thus, the antipath parts have the form

$$
\operatorname{dir}(b)=\cdots \theta_{n} \cdots \theta_{2} \theta_{1} \quad \text { and } \quad \operatorname{dir}(c)=\cdots \psi_{n} \cdots \psi_{2} \psi_{1},
$$

where $\theta_{1}=b$ and $\psi_{1}=d$ and $b, d \in Q_{1}$ are such that $b w$ and $d v$ are defined as strings.

Since $\varphi: P_{\sigma}^{\bullet} \rightsquigarrow P_{\tau}^{\bullet}$ is supported in cohomological degree $-k \leq-2$, we have the following subdiagram of the unfolded diagram for $\varphi$.

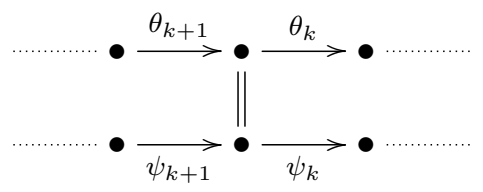


We first show that $\varphi$ is supported in degrees $-k-1$ and $-k+1$. Suppose that $\varphi$ was not supported in cohomological degree $-k+1$, then inverting $\tau$ the unfolded diagram of $\varphi$ would have the form,

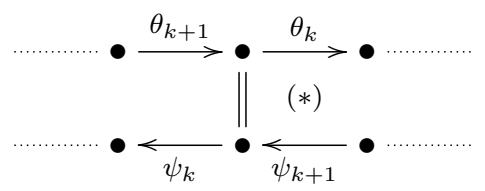

where $(*)$ corresponds to the graph map right endpoint condition (RG3) in [9, §1.4.1], whence by [1, Rem. 4.9] corresponds to a family of null-homotopic maps. Similarly, one can show that $\varphi$ is supported in cohomological degree $-k-1$. This means that we can extend the subdiagram of the unfolded diagram of $\varphi$ to the following,

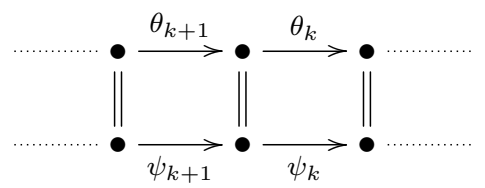

showing that $\theta_{k}=\psi_{k}$ for each $k \geq 2$. But this means that the unfolded diagram of $\varphi$ satisfies (LG3) or $(\mathrm{LG} \infty)$ (cf. $[9, \S 1.4 .1])$ and, therefore, invoking [1, Rem. 4.9] again, we see that $\varphi$ corresponds to a null-homotopic family of single and double maps. This contradicts our assumption that $\varphi$ is a quasi-graph map, therefore $\varphi$ cannot be supported in cohomological degrees smaller than -2 , as claimed.

We now consider the endpoints of a quasi-graph map $\varphi: Q_{\sigma}^{\bullet} \rightsquigarrow Q_{\tau}^{\bullet}$. Lemma 3.8 says that they must occur in degrees -1 or 0 . Recall the definition of homotopy strings or bands $\sigma$ and $\tau$ being compatibly oriented for a quasi-graph map $\varphi$ from [9, Def. 3.1]; note that if a quasi-graph map is supported in more than one degree it is automatically compatibly oriented in its unfolded form.

Lemma 3.9. Suppose the quasi-graph map $\varphi: Q_{\sigma}^{\bullet} \rightsquigarrow Q_{\tau}^{\bullet}$ has right endpoint in degree 0.

(1) The compatibly oriented unfolded diagram for $\varphi$ has the following form at the right endpoint of $\varphi$ :

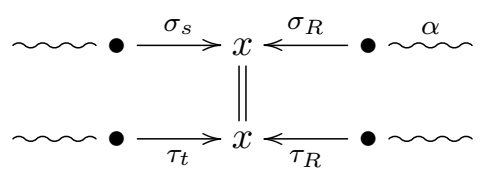

such that $\sigma_{s}, \sigma_{R} \neq \varnothing, \tau_{t}=\varnothing$ or $\tau_{t}=\sigma_{s}^{\prime} \sigma_{s}$ for some (possibly nontrivial) $\sigma_{s}^{\prime}$, and $\tau_{R}=\varnothing$ or $\tau_{R}=\sigma_{R} \sigma_{R}^{\prime}$ for some nontrivial $\sigma_{R}^{\prime}$.

(2) Write $\sigma_{R}=\bar{a}_{k} \cdots \bar{a}_{1}$ and $\sigma_{s}=b_{l} \cdots b_{1}$ for $k, l \geq 1$ and $a_{i}, b_{j} \in Q_{1}$. Then

(i) $v$ has a substring of the form

$$
\widetilde{v}=\left\{\begin{array}{l}
b_{l-1} \cdots b_{1} \bar{a}_{k} \cdots \bar{a}_{2} \quad \text { if } \sigma_{R} \text { is incident with } \operatorname{inv}(v), \\
b_{l-1} \cdots b_{1} \bar{a}_{k} \cdots \bar{a}_{1} a \quad \text { for some } a \in Q_{1} \text { otherwise; }
\end{array}\right.
$$

(ii) $w$ has a substring of the form

$$
\widetilde{w}= \begin{cases}b_{l-1} \cdots b_{1} \bar{a}_{k} \cdots \bar{a}_{1} \bar{a}^{\prime} & \text { for some } a^{\prime} \in Q_{1} \text { if } \tau_{R} \neq \varnothing, \\ b_{l-1} \cdots b_{1} \bar{a}_{k} \cdots \bar{a}_{1} & \text { otherwise. }\end{cases}
$$

Proof. (1) Since $P(x)$ sits in degree zero it must be a sink for any differential incident with it because $Q_{\sigma}^{\bullet}$ and $Q_{\tau}^{\bullet}$ are projective resolutions. If $\sigma_{s}=\varnothing$ or $\sigma_{R}=\varnothing$, then the diagram indicates a graph map endpoint and $\varphi: Q_{\sigma}^{\bullet} \rightsquigarrow Q_{\tau}^{\bullet}$ is not a quasi-graph map. Therefore, $\sigma_{s}, \sigma_{R} \neq \varnothing$. If $\tau_{R} \neq \varnothing$, the orientation of the differentials means that $\varphi$ must satisfy the quasi-graph map right endpoint condition (RQ2), whence $\tau_{R}=\sigma_{R} \sigma_{R}^{\prime}$ for some nontrivial $\sigma_{R}^{\prime}$. The statement regarding $\tau_{t}$ just lists the possible cases that may occur with the given orientation.

(2) (i) First note that $b_{l-1} \cdots b_{1}$ is a substring of $v$ by Corollary 2.12. If $\sigma_{R}$ is incident with $\operatorname{inv}(v)$ then the first statement is clear. By Remark 2.15(1), $\sigma$ cannot start with the inverse 
homotopy letter $\sigma_{R}$ unless it is incident with $\operatorname{inv}(v)$. Thus, if $\sigma_{R}$ is not incident with $\operatorname{inv}(v)$ then $\alpha$ must end with a direct homotopy letter, whose last arrow we denote by $a \in Q_{1}$, say, giving the required form for $\widetilde{v}$.

(2) (ii) We treat this in cases. Firstly, if $\tau_{t}, \tau_{R}=\varnothing$, then $Q_{\tau}^{\bullet}$ is the stalk complex $P(x)$ concentrated in degree zero. Using the form of $\sigma_{s}$ and $\sigma_{R}$ we see that $P(x) \cong M(u)$ for some string $u=q b_{l} \cdots b_{1} \bar{a}_{k} \cdots \bar{a}_{1} \bar{p}$, where $q$ is a maximal direct string and $\bar{p}$ a maximal inverse string composable with $b_{l}$ and $\bar{a}_{k}$, respectively, as strings. The claim is now clear in this case.

Now assume that $\tau_{t} \neq \varnothing$ and $\tau_{R}=\varnothing$. By (1), $\tau_{t}=\sigma_{s}^{\prime} \sigma_{s}$, where $\sigma_{s}^{\prime}$ is possibly trivial. Since $\tau_{R}=\varnothing, w$ either starts with $b_{1}$ (a direct arrow) or else $w$ has had a maximal inverse prefix removed. The former case cannot occur because $b_{1} \bar{a}_{k}$ is defined as a string, which by Corollary 2.12 would make $\tau_{R} \neq \varnothing$. Thus, by gentleness, $w=u \bar{a}_{1} w_{R}$ for some (possibly trivial) inverse string $w_{R}$. If $\bar{a}_{k} \cdots \bar{a}_{1}$ is a (possibly equal) substring of $\bar{a}_{k} w_{R}$ then $w$ contains the substring $\widetilde{w}$ as claimed. So suppose $\bar{a}_{k} w_{R}=\bar{a}_{k} \cdots \bar{a}_{i}$ for some $1<i \leq k$. Then, $\bar{a}_{k} w_{R} \bar{a}_{i-1}$ is defined as a string, again rendering $\tau_{R} \neq \varnothing$ by Corollary 2.12; a contradiction.

Suppose now that $\tau_{t}=\varnothing$ and $\tau_{R} \neq \varnothing$. Since $\tau_{t}=\varnothing, w$ ends with a direct substring which has been removed by Remark 2.15(2). By gentleness, the maximal direct suffix that has been removed is $p b_{l} \cdots b_{1}$, where again $p$ is the maximal direct path composable with $b_{l}$ as a string. Now since $\tau_{R}=\sigma_{R} \sigma_{R}^{\prime}$ is a strictly longer inverse homotopy letter than $\sigma_{R}$, it follows that $\widetilde{w}$ is a substring of $w$, where $\sigma_{R}^{\prime}=\bar{a}^{\prime} \sigma_{R}^{\prime \prime}$ for some $a^{\prime} \in Q_{1}$ and $\sigma_{R}^{\prime \prime}$ is possibly trivial.

Finally, if $\tau_{t}, \tau_{R} \neq \varnothing$, then arguing as above shows that $\widetilde{w}$ is a substring of $w$.

Lemma 3.10. Suppose the quasi-graph map $\varphi: Q_{\sigma}^{\bullet} \rightsquigarrow Q_{\tau}^{\bullet}$ has right endpoint in degree -1 .

(1) The compatibly oriented unfolded diagram for $\varphi$ has the following form at the right endpoint of $\varphi$ :

(a)

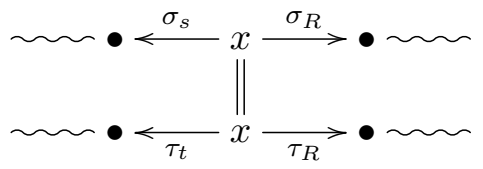

(b)

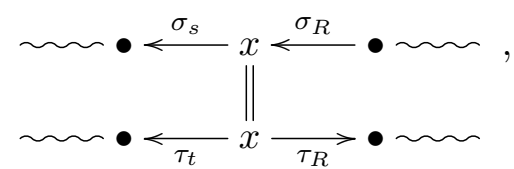

where $\tau_{t} \neq \varnothing$. In case (a), $\sigma_{s}=\varnothing$ or $\sigma_{s}=\tau_{t}^{\prime} \tau_{t}$ for some $\tau_{t}^{\prime}$ and we require $\tau_{R} \neq \varnothing$ and $\sigma_{R}=\varnothing$ or else $\sigma_{R}=\tau_{R} \tau_{R}^{\prime}$ for some nontrivial $\tau_{R}^{\prime}$. In case (b) $\sigma_{s}=\tau_{t}^{\prime} \tau_{t}$ for some $\tau_{t}^{\prime}$ and we require one of $\tau_{R} \neq \varnothing$ or $\sigma_{R} \neq \varnothing$ and if both are not empty letters then $\overline{\sigma_{R}} \tau_{R} \neq 0$. In both cases $\tau_{t}^{\prime}$ may be trivial.

(2) Write $\tau_{t}=\bar{d}_{q} \cdots \bar{d}_{1}$ and $\tau_{R}=c_{p} \cdots c_{1}$ for $k, l \geq 1$ and $c_{i}, d_{j} \in Q_{1}$. Then

(i) $v$ has a substring of the form

$$
\widetilde{v}= \begin{cases}\bar{d}_{q} \cdots \bar{d}_{2} & \text { if } \sigma_{s} \text { is incident with } \operatorname{inv}(v), \\ c_{p}-1 \cdots c_{1} c & \text { for some } c \in Q_{1} \text { if } \sigma_{s}=\varnothing \text { and } \sigma_{R} \text { is incident with } \operatorname{dir}(v), \\ \bar{d}_{q} \cdots \bar{d}_{1} c_{p} \cdots c_{1} c & \text { for some } c \in Q_{1} \text { if } \sigma_{s} \neq \varnothing \text { and } \sigma_{R} \neq \varnothing \text { is direct; }\end{cases}
$$

(ii) whas a substring of the form

$$
\widetilde{w}= \begin{cases}\bar{d}_{q} \cdots \bar{d}_{1} c_{p} \cdots c_{1} & \tau_{R} \neq \varnothing, \\ \bar{d}_{q} \cdots \bar{d}_{2} & \text { otherwise. }\end{cases}
$$

Proof. (1) There are three possible orientations for the homotopy strings $\sigma$ and $\tau$ with right endpoint in degree -1 , where in the following diagrams $x$ sits in degree -1 :

$$
(I)
$$

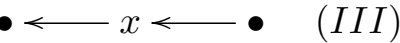

Note that the fourth possible orientation does not occur because then the corresponding string or band complex would have maximal cohomological degree -1 , contradicting Remark 2.15(4). One can check that if $\sigma$ has orientation (I) then so does $\tau$ : the other orientations produce graph map endpoint conditions (and hence null-homotopies; see [1, Rem. 4.9]), this gives case $(a)$ above. Observe that in case (a), $\tau_{t} \neq \varnothing$ and $\tau_{R} \neq \varnothing$, for otherwise we would have a graph map endpoint condition. 
If $\sigma$ has orientation (II) then $\tau$ cannot have orientation (III) because this again gives a graph map endpoint condition. If $\tau$ has orientation (II) then we may assume $\tau_{R} \neq \varnothing$ (the case $\tau_{R}=\varnothing$ is trivial can be considered as a subcase of $\tau$ having orientation (I)), in which case length $\left(\tau_{R}\right) \geq 1$. However, for degree reasons, it must be incident with $\operatorname{inv}(w)$ and hence length $\left(\tau_{R}\right)=1$. Therefore $\tau$ cannot have orientation (II). This gives us case $(b)$. Note in this case that since $x$ sits in degree $-1, \sigma_{s} \neq \varnothing$ by Remark 2.15(2); as above, $\tau_{s} \neq \varnothing$ otherwise we have a graph map endpoint condition.

When $\sigma$ has orientation (III), the unfolded diagrams are those for the dual left endpoint conditions, and can be properly stated in the dual of this lemma.

(2) (i) First observe that, in both cases, either $\sigma_{s} \neq \varnothing$ or $\sigma_{R} \neq \varnothing$ (or both) for degree reasons: if both were empty homotopy letters, $Q_{\sigma}^{\bullet}$ would be a stalk complex concentrated in degree -1 , contradicting Remark 2.15(4).

Suppose we are in case (a) of part (1). Suppose $\sigma_{s}=\varnothing$ but $\sigma_{R} \neq \varnothing$. Then $\sigma_{R}=\tau_{R} \tau_{R}^{\prime}$ for some nontrivial $\tau_{R}^{\prime}$ by the (RQ1) endpoint condition. By Remark 2.15(2), $\sigma$ cannot end with a direct homotopy letter unless it is incident with $\operatorname{dir}(v)$. Let $c \in Q_{1}$ be the final arrow of the homotopy (sub)letter $\tau_{R}^{\prime}$. Then since $\sigma_{R}$ is incident with $\operatorname{dir}(v)$, we have that $\widetilde{v}=c_{p-1} \cdots c_{1} c$ is a substring of $v$.

If $\sigma_{s} \neq \varnothing$ but $\sigma_{R}=\varnothing$, then Remark 2.15(1) shows that $\sigma_{s}$ is incident with $\operatorname{inv}(v)$, giving $\widetilde{v}=\bar{d}_{q} \cdots \bar{d}_{2}$ as a substring of $v$.

If $\sigma_{1}, \sigma_{R} \neq \varnothing$, then neither is incident with $\operatorname{dir}(v)$ or $\operatorname{inv}(v)$, in which case $\widetilde{v}=$ $\bar{d}_{q} \cdots \bar{d}_{1} c_{p} \cdots c_{1} c$, where $c \in Q_{1}$ is as above, is a substring of $v$.

Now suppose we are in case (b) of part (1). If $\sigma_{R}=\varnothing$ then using Remark 2.15(1) again we have $\sigma_{s}$ is incident with $\operatorname{inv}(v)$ and $\widetilde{v}=\bar{d}_{q} \cdots \bar{d}_{2}$ is a substring of $v$. If $\sigma_{R} \neq \varnothing$, then by Remark 2.15(5), length $\left(\sigma_{R}\right)=1$ and $\sigma_{R}$ is incident with $\operatorname{inv}(v)$, in which case $\widetilde{v}=\bar{d}_{q} \cdots \bar{d}_{2}$ is again a substring of $v$.

(2) (ii) Suppose we are in case (a) of part (1). Since $\tau_{t}, \tau_{R} \neq \varnothing$, the homotopy substring $\tau_{1} \tau_{R}$ cannot be incident with $\operatorname{dir}(w)$ nor $\operatorname{inv}(w)$ for degree reasons. Thus, $\widetilde{w}=\bar{d}_{q} \cdots \bar{d}_{1} c_{p} \cdots c_{1}$ is a substring of $w$.

Finally, suppose we are in case $(b)$ of (1). If $\tau_{R}=\varnothing$, then Remark 2.15(1) shows that $\tau_{1}$ is incident with $\operatorname{inv}(w)$, giving $\widetilde{w}=\bar{d}_{q} \cdots \bar{d}_{2}$ as a substring of $w$. If $\tau_{R} \neq \varnothing$, then as above the homotopy substring $\tau_{1} \tau_{R}$ cannot be incident with $\operatorname{dir}(w)$ nor $\operatorname{inv}(w)$. Thus, $\widetilde{w}=\bar{d}_{q} \cdots \bar{d}_{1} c_{p} \cdots c_{1}$ is a substring of $w$.

Lemmas 3.9 and 3.10 admit obvious duals for the left endpoints of quasi-graph maps.

Now applying the graphical calculus for the mapping cones of the homotopy set determined by a quasi-graph map [9, Prop. 5.2] and [10, §2] determines the middle term of the extension $Q_{\tau}^{\bullet} \rightarrow E^{\bullet} \rightarrow Q_{\sigma}^{\bullet} \rightarrow \Sigma Q_{\tau}^{\bullet}$ in $\mathrm{K}^{b,-}(\operatorname{proj}(\Lambda))$. Lemmas 3.9 and 3.10 and their duals, Theorem 2.8, together with a calculation as in the proof of Lemma 3.5 allows us to take cohomology to determine the extension $0 \rightarrow M(w) \rightarrow H^{0}\left(E^{\bullet}\right) \rightarrow M(v) \rightarrow 0$. We summarise this computation in the next proposition.

Proposition 3.11. Suppose $\varphi: Q_{\sigma}^{\bullet} \rightsquigarrow Q_{\tau}^{\bullet}$ is a quasi-graph map with the following unfolded diagram, with $t \geq 0$ and, when $t=0$ we mean a quasi-graph map supported in precisely one degree and we replace $\rho_{1}$ by $\sigma_{L}$ and $\tau_{L}$ as appropriate.

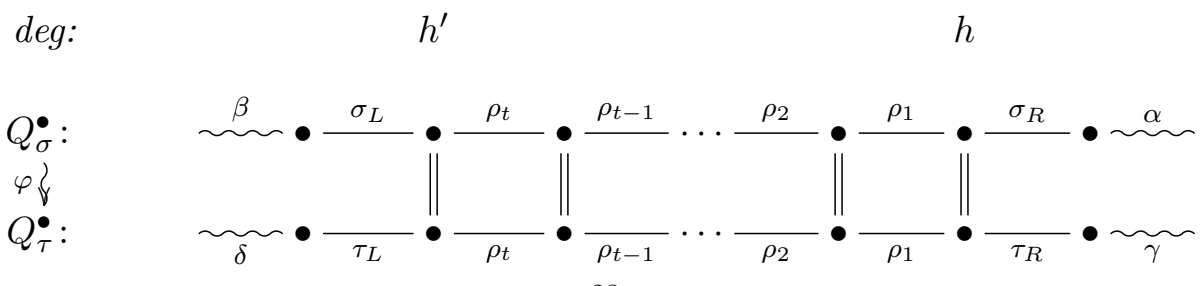


Let $f^{\bullet}: Q_{\sigma}^{\bullet} \rightarrow \Sigma Q_{\tau}^{\bullet}$ be any representative of $\varphi$, then $\Phi\left(f^{\bullet}\right)$ is an overlap extension with overlap $m=m_{L} \rho_{t-1} \cdots \rho_{2} m_{R}$, where

$$
\begin{aligned}
& m_{R}= \begin{cases}\widetilde{\rho}_{1} \bar{a}_{k} \cdots \bar{a}_{2} & \text { if } h=0 \text { and } \sigma_{R}=\bar{a}_{k} \cdots \bar{a}_{2} \text { is incident with } \operatorname{inv}(v) ; \\
\widetilde{\rho}_{1} \bar{a}_{k} \cdots \bar{a}_{1} & \text { if } h=0 \text { and } \sigma_{R}=\bar{a}_{k} \cdots \bar{a}_{1} \text { is not incident with } \operatorname{inv}(v) ; \\
\bar{d}_{q} \cdots \bar{d}_{2} & \text { if } h=-1 \text { and } \rho_{1} \neq \varnothing \text { is incident with } \operatorname{inv}(v) ; \\
\widetilde{\rho}_{1} c_{p} \cdots c_{2} & \text { if } h=-1, \rho_{1} \neq \varnothing \text { and } \sigma_{R}=c_{p} \cdots c_{1} \text { with } p>0 ;\end{cases} \\
& m=c_{p-1} \cdots c_{1} \quad \text { if } \rho_{1}=\varnothing \text { and } \sigma_{R}=c_{p} \cdots c_{1} \text { is incident with } \operatorname{dir}(v),
\end{aligned}
$$

where $a_{i}, d_{i}, c_{i} \in Q_{1}, m_{L}$ is defined dually, and

$$
\widetilde{\rho_{1}}= \begin{cases}\rho_{1} & \text { if } t \geq 1, \\ \text { the last homotopy letter of } m_{L} & \text { if } t=0 .\end{cases}
$$

Remark 3.12. The analysis concerning quasi-graph maps above leading to Proposition 3.11 only concerns the endpoints of the overlap defining a quasi-graph map in the unfolded diagram. As such, the length of the overlap is not relevant for the argument. In particular, this means that when one (or both) of $v$ or $w$ is a band, and thus $\sigma$ or $\tau$ is a homotopy band, we are able to get quasi-graph maps whose overlaps are longer than at least one of the bands, but this does not affect the computation carried out in Proposition 3.11.

3.3. Singleton maps. As before, throughout this subsection $\sigma=\pi(v)$ and $\tau=\pi(w)$ for some strings or bands $v$ and $w$. We now examine the kinds of extensions that arise from singleton (single and double) maps $f^{\bullet}: Q_{\sigma}^{\bullet} \rightarrow \Sigma Q_{\tau}^{\bullet}$. We first note that singleton double maps never occur as morphisms between projective resolutions of modules.

Lemma 3.13. There are no singleton double maps $f^{\bullet}: Q_{\sigma}^{\bullet} \rightarrow \Sigma Q_{\tau}^{\bullet}$.

Proof. By definition, the unfolded diagram of a singleton double map has the form

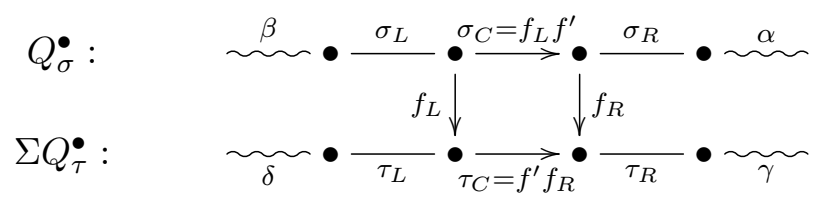

where $f_{L}, f^{\prime}$ and $f_{R}$ are nontrivial. By Remark 2.15(5), length $\left(\sigma_{C}\right)>1$ and length $\left(\tau_{C}\right)>1$. In particular, since $\sigma$ is a homotopy string or band corresponding to a projective resolution, $\sigma_{C}$ is a homotopy letter occurring between degrees -1 and 0 . On the other hand, $\tau$ is also a homotopy string or band corresponding to a projective resolution, but $\Sigma Q_{\tau}^{\bullet}$ has been shifted, whence $\tau_{C}$ must be a homotopy letter occurring between degrees -2 and -1 . Hence there are no such maps.

Recall the notation and unfolded diagram for a singleton single map $f^{\bullet}: Q_{\sigma}^{\bullet} \rightarrow \Sigma Q_{\tau}^{\bullet}$ from Section 1.4.2(4). Throughout this section, whenever $\sigma_{R} \neq \varnothing$ or $\tau_{R} \neq \varnothing$ in (4) then since $f_{R}$ and $f_{L}$ are direct strings, we can assume, without loss of generality, that $f_{R} \in Q_{1}$ and $f_{L} \in Q_{1}$, respectively.

Lemma 3.14. Suppose $f^{\bullet}: Q_{\sigma}^{\bullet} \rightarrow \Sigma Q_{\tau}^{\bullet}$ as a singleton single map with single component $f=$ $f_{n} \cdots f_{1}$. Then the component $f$ occurs in cohomological degree -1 .

Proof. Suppose $f^{\bullet}$ is supported in cohomological degree $d$. Since $Q_{\tau}^{\bullet}$ is a projective resolution, $\Sigma Q_{\tau}^{\bullet}$ attains its maximal degree in degree -1 , thus $d \leq-1$. By Remark 2.15(5), if in (4) either $\sigma_{R} \neq \varnothing$ or $\tau_{R} \neq \varnothing$ then $d=-1$. So assume $\sigma_{R}, \tau_{R}=\varnothing$ and $d<-1$. By Corollary 2.12, since $\tau_{L}$ is the endpoint of a homotopy string occurring in degree $d$ it must be inverse (otherwise there would be nontrivial cohomology in degree $d$, contradicting the fact that $\Sigma Q_{\tau}^{\bullet}$ is a (shifted) projective resolution). Moreover, for degree reasons, $\tau_{L}$ must be the first homotopy letter of $\operatorname{inv}(w)$. Writing $\tau_{L}=\bar{b}_{l} \cdots \bar{b}_{1}$ for some $b_{i} \in Q_{1}, i=1, \ldots, l$, the definition of single maps gives us that $\bar{b}_{1} \bar{f}_{1}=0$. This contradicts the fact that $\operatorname{inv}(w)$ is the longest inverse antipath incident with $w^{\prime}$ (see Corollary 2.12). Therefore, $d=-1$, as claimed. 
Corollary 3.15. Suppose $f^{\bullet}: Q_{\sigma}^{\bullet} \rightarrow \Sigma Q_{\tau}^{\bullet}$ is a singleton single map. In the unfolded diagram (4) in Section 1.4.2, $\tau_{L}$ must be a direct homotopy letter or $\tau_{L}=\varnothing$.

Proof. Since $\Sigma Q_{\tau}^{\bullet}$ attains its maximal cohomological degree in degree -1 and $f^{\bullet}$ is supported in degree -1 by Lemma $3.14, \tau_{L}$ cannot be inverse.

Corollary 3.15 allows us to further specialise the setup in Section 1.4.2(4) in the statement of the next proposition.

Proposition 3.16. Suppose $f^{\bullet}: Q_{\sigma}^{\bullet} \rightarrow \Sigma Q_{\tau}^{\bullet}$ is a singleton single map with single component $f=f_{n} \cdots f_{1}$. Write $\tau_{L}=b_{l} \cdots b_{1}$ with $b_{i} \in Q_{1}$ for $i=1, \ldots, l$. Whenever $\sigma_{L}$ is an inverse homotopy letter we shall write $\sigma_{L}=\bar{a}_{k} \cdots \bar{a}_{1}$, where $a_{i} \in Q_{1}$ for $i=1, \cdots, k$ with $k \geq 1$.

(1) If $\sigma_{R}=\varnothing$ then $\tau_{R}=\varnothing$ and $\sigma_{L}$ is inverse. The corresponding extension $\Phi\left(f^{\bullet}\right) \in$ $\operatorname{Ext}_{\Lambda}^{1}(M(\bar{v}), M(w))$ is an arrow extension given by $a_{1}$, i.e. $\Phi\left(f^{\bullet}\right)$ gives rise to an extension of $M(w)$ by $M(\bar{v})$ with middle term $M(u)$ where $u=w a_{1} \bar{v}$.

Suppose $\sigma_{R} \neq \varnothing$. If $\sigma_{R}$ is not incident with $\operatorname{dir}(v)$ then $\sigma_{L}$ is inverse and we have:

(2) If $\tau_{R}=\varnothing$ then the corresponding extension $\Phi\left(f^{\bullet}\right) \in \operatorname{Ext}_{\Lambda}^{1}(M(\bar{v}), M(w))$ is an overlap extension whose middle term is given by

$m=\bar{f}_{1} \cdots \bar{f}_{n-1}, A=\varnothing, B=f_{R}, C=f_{n}$ and $D=b_{1} \quad$ if $\sigma_{R}$ is incident with $\operatorname{dir}(v)$;

$m=\bar{f}_{1} \cdots \bar{f}_{n}, A=a_{1}, B=f_{R}, C=\varnothing$ and $D=b_{1} \quad$ otherwise.

(3) If $\tau_{R} \neq \varnothing$ then the corresponding extension $\Phi\left(f^{\bullet}\right) \in \operatorname{Ext}_{\Lambda}^{1}(M(\bar{v}), M(w))$ is an overlap extension which, when $\sigma_{R}$ is incident with $\operatorname{dir}(v)$, has its middle term given by,

$$
\begin{aligned}
& m=\bar{f}_{1} \cdots \bar{f}_{n-1}, \quad A=\varnothing, \quad B=f_{R}, \quad C=f_{n} \quad \text { and } \quad D=c_{1} \quad \text { if } \tau_{L}=\varnothing ; \\
& m=\bar{f}_{1} \cdots \bar{f}_{n-1}, \quad A=\varnothing, \quad B=f_{R}, \quad C=f_{n} \quad \text { and } \quad D=b_{1} \quad \text { if } \tau_{L} \neq \varnothing,
\end{aligned}
$$

and is an overlap extension which, when $\sigma_{R}$ is not incident with $\operatorname{dir}(v)$, has its middle term given by,

$$
\begin{aligned}
& m=\bar{f}_{1} \cdots \bar{f}_{n}, \quad A=a_{1}, \quad B=f_{R}, \quad C=\varnothing \quad \text { and } \quad D=c_{1} \quad \text { if } \tau_{L}=\varnothing ; \\
& m=\bar{f}_{1} \cdots \bar{f}_{n}, \quad A=a_{1}, \quad B=f_{R}, \quad C=\varnothing \quad \text { and } \quad D=b_{1} \quad \text { if } \tau_{L} \neq \varnothing .
\end{aligned}
$$

In case (3) of the proof below, we do an example of a computation as in Lemma 3.5 for an overlap extension. An example computation for an arrow extension is done in the proof of Lemma 3.5, and we refer the reader to Figure 3 for a schematic of such a computation.

Proof. (1) First note that $\sigma_{L}$ is inverse, since if it were direct or empty $Q_{\sigma}^{\bullet}$ would have nontrivial cohomology in degree -1 , contradicting the fact that it is a projective resolution. Therefore, $\sigma_{L}=\bar{a}_{k} \cdots \bar{a}_{1}$ with $a_{i} \in Q_{1}$ for $i=1, \ldots, k$ for some $k \geq 1$. Moreover, $\sigma_{L}$ is the start of $\operatorname{inv}(v)$, for otherwise $\sigma$ would start in degree 0 after the removal of a maximal inverse prefix. It follows that $v$ starts with the inverse substring $\bar{a}_{k} \cdots \bar{a}_{2}$, whence $\bar{v}$ ends with the direct substring $a_{2} \cdots a_{k}$.

Consider the local subquiver of $Q$, where, without loss of generality, we assume $f_{L} \in Q_{1}$,

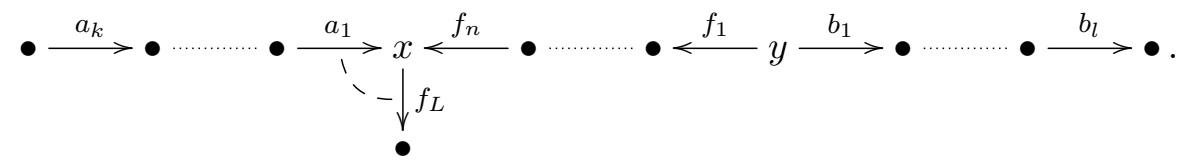

If $\tau_{R} \neq \varnothing$ then $\bar{a}_{1} \bar{f}_{L}=0$, contradicting the fact that $\sigma_{L}$ is the start of $\operatorname{inv}(v)$. Thus, $\tau_{R}=\varnothing$.

Since $f$ is not a subletter of $\tau_{L}$ or vice versa we must have $f_{1} \neq b_{1}$ and $b_{1} \bar{f}_{1}$ is defined as a string. This means that a maximal inverse prefix, whose last (inverse) arrow is $\bar{f}_{1}$, has been removed from $w$ in the computation of $\tau=\pi(w)$ for otherwise $\tau_{R} \neq \varnothing$. We claim that $\bar{f}$ is precisely the maximal inverse prefix that has been removed. Clearly, the maximal inverse prefix cannot be a proper substring of $\bar{f}$ for the computation of $\tau=\pi(w)$ in Corollary 2.12 would require us to compose this with $w$ giving $\tau_{R} \neq \varnothing$. However, if $\bar{f}$ were a proper substring of the 
maximal inverse prefix then there would be an arrow $f_{n+1} \in Q_{1}$ such that $\bar{a}_{1} \bar{f}_{n+1}=0$ giving us a contradiction as above. Therefore, $w$ starts with the substring $\tau_{L} \bar{f}$. Applying [9, Thm. 3.2], Theorem 2.8 and a computation as in Lemma 3.5 shows that that $\Phi\left(f^{\bullet}\right)$ gives an arrow extension corresponding to the arrow $a_{1}$ with middle term $M(u)$, where $u=w a_{1} \bar{v}$.

Suppose that $\sigma_{R} \neq \varnothing$. If $\sigma_{R}$ is not incident with $\operatorname{dir}(v)$ then by Remark 2.15(1), $\sigma_{L} \neq \varnothing$ and is inverse and we write $\sigma_{L}=\bar{a}_{k} \cdots \bar{a}_{1}$, where $a_{i} \in Q_{1}$ for $i=1, \ldots, k$ with $k \geq 1$.

(2) Suppose that $\tau_{R}=\varnothing$. First observe that, by Corollary 2.12, $w$ has a substring of the form $b_{l-1} \cdots b_{1} \bar{f}_{1} \cdots \bar{f}_{n}$. If $\sigma_{R}$ is incident with $\operatorname{dir}(v)$ (in which case so is $\sigma_{L}$ regardless of whether it is empty), then $v$ ends with a substring $f_{n-1} \cdots f_{1} f_{R}$, i.e. $\bar{v}$ starts with a substring $\bar{f}_{R} \bar{f}_{1} \cdots \bar{f}_{n-1}$, by Corollary 2.12. Applying [9, Thm. 3.2], taking cohomology using Theorem 2.8 and a calculation as in Lemma 3.5 then gives us an overlap extension between $M(w)$ and $M(\bar{v})$ :

$$
m=\bar{f}_{1} \cdots \bar{f}_{n-1}, \quad A=\varnothing, \quad B=f_{R}, \quad C=f_{n} \quad \text { and } \quad D=b_{1} .
$$

Now suppose $\sigma_{R}$ is not incident with $\operatorname{dir}(v)$. By Corollary $2.12 v$ has a substring $\sigma_{L} f_{n} \cdots f_{1} f_{R}$, i.e. $\bar{v}$ has a substring $\bar{f}_{R} \bar{f}_{1} \cdots \bar{f}_{n} \bar{\sigma}_{L}$. Again applying [9, Thm. 3.2], Theorem 2.8 and a calculation as in Lemma 3.5 gives us the following overlap extension between $M(w)$ and $M(\overline{(} v))$ :

$$
m=\bar{f}_{1} \cdots \bar{f}_{n}, \quad A=a_{1}, \quad B=f_{R}, \quad C=\varnothing \quad \text { and } \quad D=b_{1} .
$$

(3) Suppose that $\tau_{R} \neq \varnothing$. First we assume $\sigma_{R}$ is incident with $\operatorname{dir}(v)$, whence $\bar{v}$ starts with the substring $\bar{f}_{R} \bar{f}_{1} \cdots \bar{f}_{n-1}$ as above. If $\tau_{L}=\varnothing$, then, by Corollary 2.12, $w$ ends with a substring $c_{t} \cdots c_{1} \bar{f}_{1} \cdots \bar{f}_{n} \bar{f}_{L}$, where $c_{i} \in Q_{1}$ for $i=1, \cdots, t$ and $t \geq 0$. In this case the application of [9, Thm. 3.2], Theorem 2.8 and a calculation as in Lemma 3.5, which we sketch below, gives us the following overlap extension between $M(w)$ and $M(\bar{v})$ :

$$
m=\bar{f}_{1} \cdots \bar{f}_{n-1}, \quad A=\varnothing, \quad B=f_{R}, \quad C=f_{n} \quad \text { and } \quad D=c_{1} .
$$

We now sketch the calculation as in Lemma 3.5 for this case. The unfolded diagrams of the morphisms occurring in the mapping cone triangle of [9, Thm. 3.2] are:

$$
\begin{gathered}
Q_{\tau}^{\bullet}: \\
g_{i}^{\bullet} \downarrow \\
E_{1}^{\bullet}: \\
h_{i}^{\bullet} \\
Q_{\sigma}^{\bullet}:
\end{gathered}
$$


Figure 4 below shows the calculation of the induced maps in cohomology: it is clear that they are the canonical maps in the resulting overlap extension. If $\tau_{L} \neq \varnothing$, then $w$ has a substring $b_{l-1} \cdots b_{1} \bar{f}_{1} \cdots \bar{f}_{n} \bar{f}_{L}$. Applying [9, Thm. 3.2] and Theorem 2.8 gives us the following overlap extension between $M(w)$ and $M(\bar{v})$ :

$$
m=\bar{f}_{1} \cdots \bar{f}_{n-1}, \quad A=\varnothing, \quad B=f_{R}, \quad C=f_{n} \quad \text { and } \quad D=b_{1} .
$$

Now assume that $\sigma_{R}$ is not incident with $\operatorname{dir}(v)$, whence $\bar{v}$ has a substring $\bar{f}_{R} \bar{f}_{1} \cdots \bar{f}_{n}{\overline{\sigma_{L}}}_{\text {as }}$ above. Using the calculations of substrings of $w$ for $\tau_{L}=\varnothing$ and $\tau_{L} \neq \varnothing$ above respectively, and the application of [9, Thm. 3.2], Theorem 2.8 and a calculation as in Lemma 3.5 gives us the following overlap extensions between $M(w)$ and $M(\bar{v})$ :

$$
\begin{aligned}
& m=\bar{f}_{1} \cdots \bar{f}_{n}, \quad A=a_{1}, \quad B=f_{R}, \quad C=\varnothing \quad \text { and } \quad D=c_{1} \quad \text { if } \tau_{L}=\varnothing ; \\
& m=\bar{f}_{1} \cdots \bar{f}_{n}, \quad A=a_{1}, \quad B=f_{R}, \quad C=\varnothing \quad \text { and } \quad D=b_{1} \quad \text { if } \tau_{L} \neq \varnothing .
\end{aligned}
$$

\section{SurJectivity of $\Phi$ onto overlap AND ARrow EXtensions}

In this section, we use the combinatorics of an overlap or arrow extension to show that the isomorphism $\Phi: \operatorname{Hom}_{\mathrm{K}^{b,-}(\operatorname{proj}(\Lambda))}\left(Q_{\pi(v)}^{\bullet}, \Sigma Q_{\pi(w)}^{\bullet}\right) \rightarrow \operatorname{Ext}_{\Lambda}^{1}(M(v), M(w))$ restricts to a surjection,

$$
\Phi:\left\{\begin{array}{l}
\text { standard basis elements of } \\
\operatorname{Hom}_{\mathrm{K}^{b,-}(\operatorname{proj}(\Lambda))}\left(Q_{\pi(v)}^{\bullet}, \Sigma Q_{\pi(w)}^{\bullet}\right)
\end{array}\right\} \rightarrow\left\{\begin{array}{l}
\text { overlap and arrow extensions } \\
\eta \in \operatorname{Ext}_{\Lambda}^{1}(M(v), M(w))
\end{array}\right\} .
$$



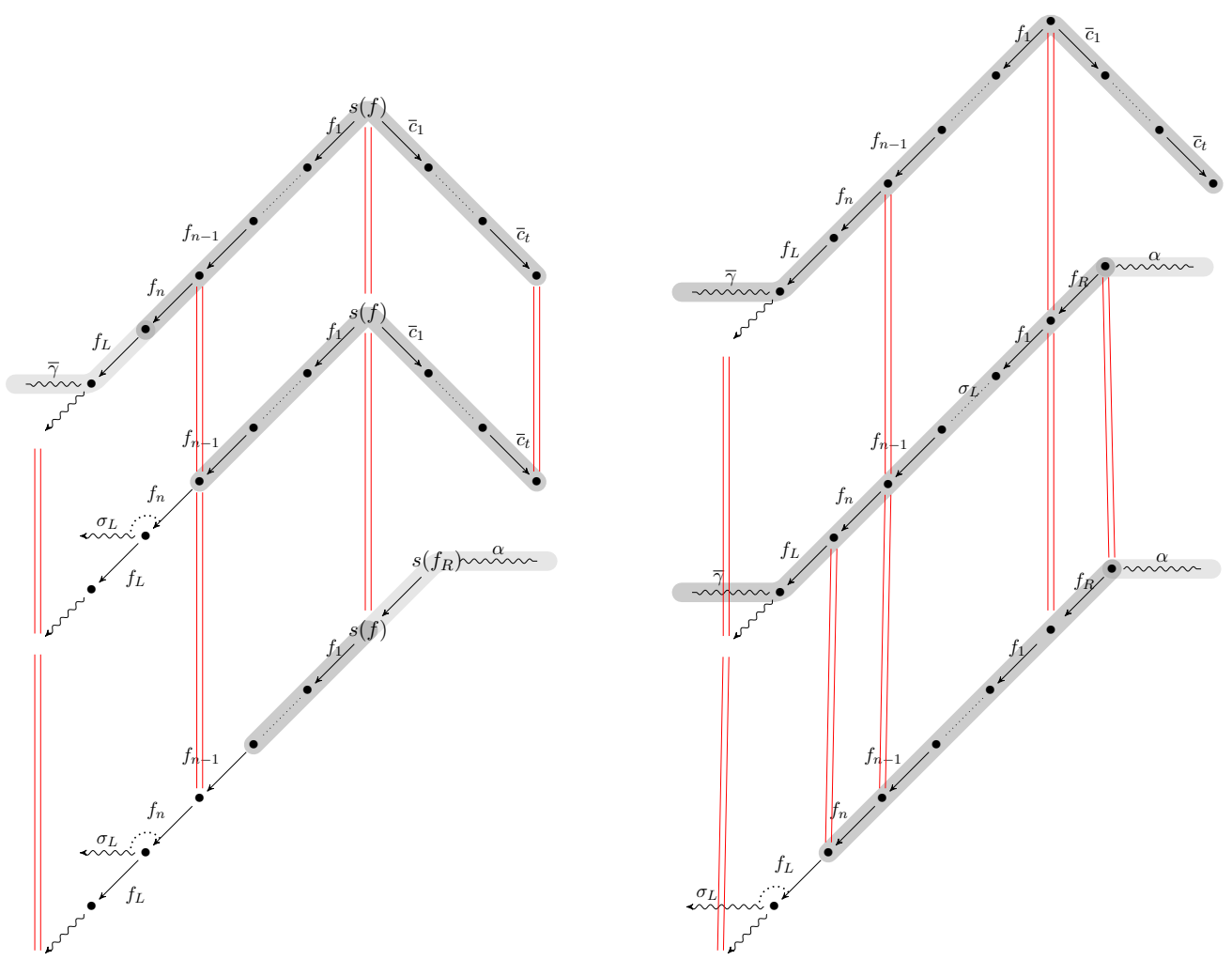

Figure 4 . The diagram of the left shows the calculation of $g_{1}^{\bullet}: Q_{\tau}^{\bullet} \rightarrow E_{1}^{\bullet}$ and $h_{1}^{\bullet}: E_{1}^{\bullet} \rightarrow Q_{\sigma}^{\bullet}$, that on the right shows the calculation of $g_{2}^{\bullet}: Q_{\tau}^{\bullet} \rightarrow E_{2}^{\bullet}$ and $h_{2}^{\bullet}: E_{2}^{\bullet} \rightarrow Q_{\sigma}^{\bullet}$ in the case (3) of the proof of Proposition 3.16.

4.1. Overlap extensions. Throughout this section we shall have the following setup.

Setup 4.1. Let $v$ and $w$ be strings or bands and $\pi(v)$ and $\pi(w)$ be the corresponding homotopy strings or bands of their projective resolutions.

Suppose $0 \neq \eta \in \operatorname{Ext}_{\Lambda}^{1}(M(v), M(w))$ is an overlap extension corresponding to the decompositions $v=v_{L} \bar{B} m A v_{R}$ and $w=w_{L} D m \bar{C} w_{R}$. We consider $m$ and decompose it into its homotopy letters $m=\mu_{l} \cdots \mu_{1}$ with $l \geq 0$. When $l=0, m$ is a trivial string, i.e. $m=1_{x}$ for some $x \in Q_{0}$ and we call it a trivial overlap. If $l=1$, we say $m$ is a direct or inverse overlap. If $l>1$, we say that $m$ is a zigzag overlap.

4.1.1. Zigzag overlaps. We start with the zigzag overlap case.

Lemma 4.2. Suppose in Setup 4.1, the string $m$ is a zigzag overlap. Then the map $f: M(w) \rightarrow$ $M(v)$ associated with this decomposition induces a graph map $f^{\bullet}: Q_{\pi(w)}^{\bullet} \rightarrow Q_{\pi(v)}^{\bullet}$ of homotopy string or band complexes such that $H^{0}\left(f^{\bullet}\right)=f$.

Proof. We first show that the given decomposition induces a graph map $Q_{\pi(w)}^{\bullet} \rightarrow Q_{\pi(v)}^{\bullet}$. It is sufficient only to consider the endpoints of the map, as determined by the decomposition. We consider only the right endpoints; the analysis for left endpoints is analogous.

Before breaking the argument up into a case analysis, first note that one of $A$ and $\bar{C}$ must exist (i.e. be nonempty) since $\eta$ is a non-split extension. By gentleness, if both $A$ and $\bar{C}$ exist we must have $C A=0$.

Case: $\mu_{1}$ is a direct homotopy letter.

By Corollary 2.12, the homotopy string or band $\pi(w)$ has the following form:

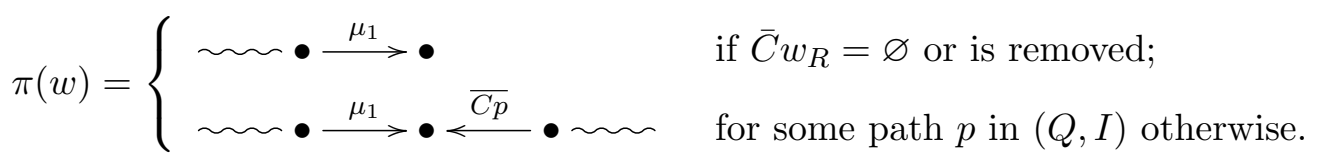


Similarly, the homotopy string or band $\pi(v)$ has the following form:

$$
\pi(v)= \begin{cases}\left.\sim \bullet \frac{\mu_{1}}{\longrightarrow} \bullet<\frac{\bar{C}}{\sim} \bullet \sim ⿴ 囗 ⿱ 一 一\right) & \text { if } A=\varnothing ; \\ \sim \stackrel{\mu_{1} A q}{\longrightarrow} \bullet \sim \quad \text { for some path } q \text { in }(Q, I) \text { otherwise. }\end{cases}
$$

Combining these, we get the following unfolded diagrams of graph map right endpoint conditions, showing the claim in this case.
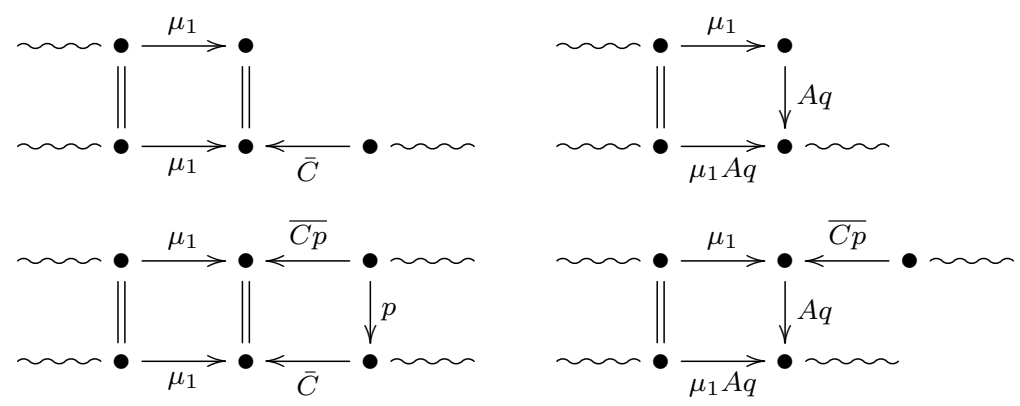

Case: $\mu_{1}$ is an inverse homotopy letter.

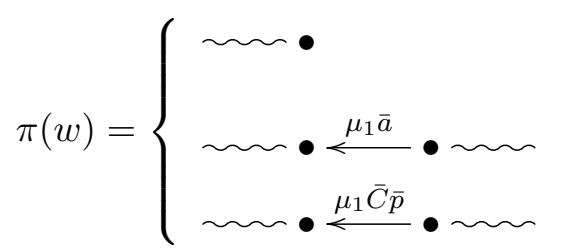

if $\bar{C}=\varnothing$ or $\bar{C} w_{R}$ is inverse, and there is no $a \in Q_{1}$ with $w \bar{a}$ defined as a string;

if $\bar{C}=\varnothing$ and there exists $a \in Q_{1}$ with $\mu_{1} \bar{a}$ a string;

for some (possibly trivial) path $p$ in $(Q, I)$, otherwise,

where the homotopy string in the first case starts with $\mu_{2}$ if it exists, or a single projective or the start of an antipath otherwise. Similarly, the homotopy string or band $\pi(v)$ has the following form:

$$
\pi(v)= \begin{cases}\sim \bullet<\frac{\mu_{1}}{\sim} \bullet \stackrel{A q}{\longrightarrow} \bullet \sim \quad \text { for some path } q \text { in }(Q, I) \text { if } A \neq \varnothing ; \\ \sim \text { if } A=\varnothing .\end{cases}
$$

We leave it to the reader to match up the various forms of the projective resolutions and confirm that they give rise to graph map right endpoint conditions as above.

Now examining the components of $f^{\bullet}: Q_{\pi(w)}^{\bullet} \rightarrow Q_{\pi(v)}^{\bullet}$ consisting of identity maps between indecomposable projective modules and following these maps through a calculation of the kind in Lemma 3.5 shows that the $H^{0}\left(f^{\bullet}\right)=f: M(w) \rightarrow M(v)$, i.e. $f^{\bullet}$ is indeed induced from $f$.

Applying Remark 1.8 we get the following corollary.

Corollary 4.3. Keep the setup as in Lemma 4.2. The map $f: M(w) \rightarrow M(v)$ induces a quasigraph map $\varphi: Q_{\pi(v)}^{\bullet} \rightsquigarrow Q_{\pi(w)}^{\bullet}$ of homotopy string or band complexes, and hence a homotopy family of maps $Q_{\pi(v)}^{\bullet} \rightarrow \Sigma Q_{\pi(w)}^{\bullet}$.

Let $g^{\bullet}: Q_{\pi(v)}^{\bullet} \rightarrow \Sigma Q_{\pi(w)}^{\bullet}$ be a representative of the homotopy family of single or double maps defined by the quasi-graph map $\varphi: Q_{\pi(v)}^{\bullet} \rightsquigarrow Q_{\pi(w)}^{\bullet}$ obtained in Corollary 4.3 above. Then, by Proposition 3.11 one obtains $\Phi\left(g^{\bullet}\right)=\eta$.

4.1.2. Direct or inverse overlaps. Here we consider the case of Setup 4.1 in which $m$ is a direct overlap; the case that $m$ is an inverse overlap is analogous. As in previous sections $\sigma=\pi(v)$ and $\tau=\pi(w)$. Again, we use the combinatorics of the overlap to define a map $g^{\bullet}: Q_{\pi(v)}^{\bullet} \rightarrow \Sigma Q_{\pi(w)}^{\bullet}$ such that $\Phi\left(g^{\bullet}\right)=\eta$. In this case, $g^{\bullet}$ is either a singleton single map or a representative of a homotopy family of maps defined by a quasi-graph map $\varphi: Q_{\pi(v)}^{\bullet} \rightsquigarrow Q_{\pi(w)}^{\bullet}$.

In the following we do a detailed analysis of the different types of standard basis maps which are induced by the different possible forms the strings $v$ and $w$ can take. We present the 
results by grouping the different cases giving rise to the same type of standard basis element in $\operatorname{Hom}_{\mathrm{K}^{b,-}(\operatorname{proj}(\Lambda))}\left(Q_{\pi(v)}^{\bullet}, \Sigma Q_{\pi(w)}^{\bullet}\right)$

Case: $g^{\bullet}: Q_{\pi(v)}^{\bullet} \rightarrow \Sigma Q_{\pi(w)}^{\bullet}$ is a singleton single map.

The unfolded diagram of the singleton single map is one of the diagrams below; we explain in which cases they arise. In each case the precise description of $\tau_{R}$ is irrelevant, we note only that in each case it is necessarily empty or an inverse homotopy letter not containing $m$ as a substring, or vice versa.
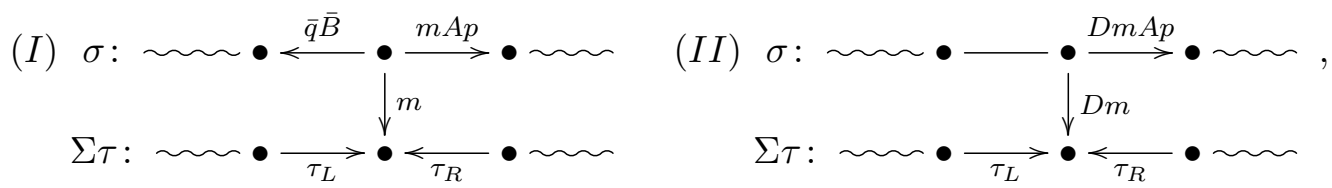

where $p$ and $q$ are (possibly trivial) paths in $(Q, I)$. Diagram (I) occurs precisely when both $A \neq \varnothing$ and $\bar{B} \neq \varnothing$ : the pertinent part of the projective resolution of $M(v)$ has this form by Corollary 2.12. Now, applying Corollary 2.12 to $w$ we see that,

$$
\tau_{L}= \begin{cases}d m & \text { if } D=\varnothing \text { but there exists } d \in Q_{1} \text { with } d m \text { defined as a string; } \\
q^{\prime} D m & \text { for some (possibly trivial) path } q^{\prime} \text { in }(Q, I) \text { if } D \neq \varnothing \text { and } w_{L} \text { is not direct or } \\
\varnothing & \begin{array}{l}
w_{L} D \text { is direct and there exists } d \in Q_{1} \text { with } d w \text { defined as a string; } \\
\text { otherwise }
\end{array}\end{cases}
$$

Diagram (II) occurs in the case that $A \neq \varnothing$ but $\bar{B}=\varnothing$; in this case to avoid $\eta$ being a split extension we must have $D \neq \varnothing$. In this case we have

$$
\tau_{L}= \begin{cases}\varnothing & \text { if } w_{L} D \text { is direct and there exists no } d \in Q_{1} \text { with } d w \text { defined as a string; } \\ q^{\prime} D m & \text { for some nontrivial path } q^{\prime} \text { in }(Q, I) \text { if the first letter of } w_{L} \text { is not inverse and } \\ & \text { we are not in the case above. }\end{cases}
$$

Note that in the case above when the first letter of $w_{L}$ is inverse, we do not get a singleton single map, hence this case is included in this argument but is treated in the next case below. In each case it is straightforward to verify that the diagram defines a singleton single map. One now applies Proposition 3.16 to see that $\Phi\left(g^{\bullet}\right)=\eta$.

Case: $g^{\bullet}: Q_{\pi(v)}^{\bullet} \rightarrow \Sigma Q_{\pi(w)}^{\bullet}$ is a representative of a homotopy family determined by a quasi-graph $\operatorname{map} \varphi: Q_{\pi(v)}^{\bullet} \rightsquigarrow Q_{\pi(w)}^{\bullet}$.

We actually check that we get a graph map $f^{\bullet}: Q_{\pi(w)}^{\bullet} \rightarrow Q_{\pi(v)}^{\bullet}$ in the opposite direction and apply Remark 1.8.

In the case that $A \neq \varnothing$ but $\bar{B}=\varnothing$, and the first letter of $w_{L}$ is inverse, i.e. the one case excluded in treating diagram (II) above, then we get the following graph map, in which $p$ is some (possibly trivial) path in $(Q, I)$.

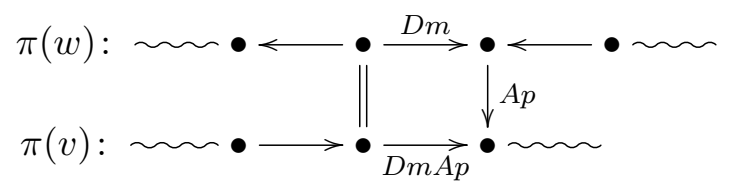

Now suppose $A=\varnothing$, whence $\bar{C} \neq \varnothing$. The overlap data gives rise to a graph map with the unfolded diagram,

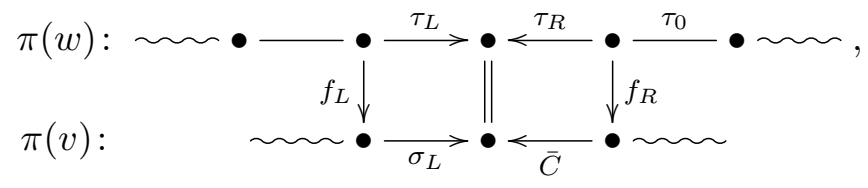




\begin{tabular}{|c|c|c|c|c|c|}
\hline$f_{L}$ & $\sigma_{L}=m$ & $\sigma_{L}=D m$ & & $f_{R}$ & $\sigma_{R}=\bar{C}$ \\
\hline$\tau_{L}=\varnothing$ & $\varnothing$ & $\varnothing$ & and & $\tau_{R}=\bar{C} \bar{p}$ & $p$ \\
\hline$\tau_{L}=q D m$ & $q D$ & $q$ & & $\tau_{R}=\varnothing$ & $\varnothing$ \\
\hline
\end{tabular}

TABLE 1. Left: the value of $f_{L}$ in each case; right: the value of $f_{R}$ in each case.

in which,

$$
\begin{aligned}
\sigma_{L} & = \begin{cases}m & \text { if } \bar{B} \neq \varnothing ; \\
D m & \text { if } \bar{B}=\varnothing ;\end{cases} \\
\tau_{R} & = \begin{cases}\bar{C} \bar{p} & \text { if } \bar{C} w_{R} \text { is not removed when computing } \pi(w) ; \\
\varnothing & \text { otherwise; }\end{cases} \\
\tau_{L} & = \begin{cases}\varnothing & \text { if } w_{L} D m \text { is removed when computing } \pi(w) ; \\
q D m & \text { otherwise }\end{cases}
\end{aligned}
$$

where $p$ is some (possibly trivial) path in $(Q, I)$ and $q$ is either the (possibly trivial) maximal direct prefix of $w_{L}$ if $w_{L} \neq \varnothing$ is not a direct string, and $q=d w_{L}$ for some $d \in Q_{1}$ with $d w_{L}$ defined as a string otherwise; in this second case, $w_{L}$ is also possibly empty. The values of $\left(f_{L}, f_{R}\right)$ in each case are recorded in Table 1

4.1.3. Trivial overlaps. We finally turn our attention to trivial overlaps. Suppose $m=1_{x}$ for some $x \in Q_{0}$. In this case, we fix the orientation of our strings and bands by requiring, whenever the relevant arrows exist, that $C B \neq 0$ and $D A \neq 0$. We again describe in each case how the combinatorics of the overlap can be used to construct a standard basis map $g^{\bullet}: Q_{\pi(v)}^{\bullet} \rightarrow Q_{\pi(w)}^{\bullet}$ such that $\Phi\left(g^{\bullet}\right)=\eta$.

Case: $g^{\bullet}: Q_{\pi(v)}^{\bullet} \rightarrow \Sigma Q_{\pi(w)}^{\bullet}$ is a graph map supported in one degree.

This is simply a degeneration of diagram (I) in the singleton single map case of Section 4.1.2, where instead $m=1_{x}$ for some vertex $x \in Q_{0}$, i.e. providing a graph map concentrated in one degree. Applying Lemma 3.6 we get $\Phi\left(g^{\bullet}\right)=\eta$.

Case: $g^{\bullet}: Q_{\pi(v)}^{\bullet} \rightarrow \Sigma Q_{\pi(w)}^{\bullet}$ is a singleton single map.

If $A=\varnothing$ and $B \neq \varnothing$, in which case $\bar{C} \neq \varnothing$, then by Corollary 2.12, the homotopy string $\pi(v)$ has the form

$$
\pi(v): \sim \bullet \stackrel{\bar{q} \bar{B} \bar{C}}{<} \bullet<\frac{\sigma_{R}}{<} \bullet \sim,
$$

where $\sigma_{R}$ may be an empty homotopy letter. Similarly, the homotopy string $\pi(w)$ has the form

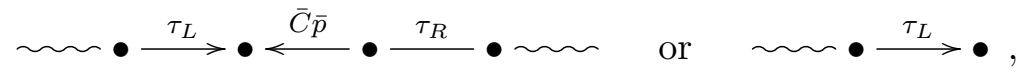

where $p$ is a (possibly trivial) path in $(Q, I)$, and $\tau_{L}$ and $\tau_{R}$ are possibly empty homotopy letters. The form of $\tau_{L}$ depends on the form of the substring $w_{L} D$, but is not relevant for the description of the map. The second case occurs when $w$ starts with $\bar{C}$ and we fall in case (3) or (4) of Corollary 2.12. In the case that $p$ is nontrivial, we get the unfolded diagram on the left below. In the case that $\pi(w)$ starts with $\tau_{L}$, we get the unfolded diagram on the right. In both cases we get a singleton single map.
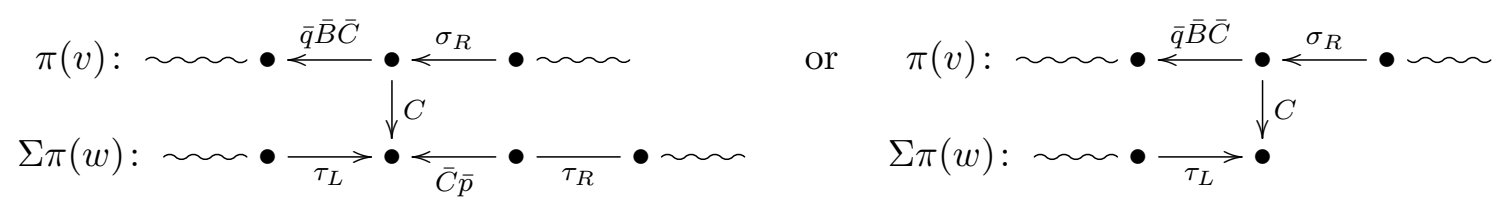

The case that $p$ is trivial gives rise to a quasi-graph map, which is dealt with below. There are obvious dual considerations when $A \neq \varnothing$ and $B=\varnothing$. Now apply Proposition 3.16. 
Case: $g^{\bullet}: Q_{\pi(v)}^{\bullet} \rightarrow \Sigma Q_{\pi(w)}^{\bullet}$ is a representative of a homotopy family determined by a quasi-graph $\operatorname{map} \varphi: Q_{\pi(v)}^{\bullet} \rightsquigarrow Q_{\pi(w)}^{\bullet}$.

In the case that $A=\varnothing$ but $B \neq \varnothing$ above, in which the path $p$ occurring in the homotopy string $\pi(w)$ is trivial, we must have that $\tau_{R} \neq \varnothing$ and is direct by Corollary 2.12. This gives rise to a graph map $f^{\bullet}: Q_{\pi(w)}^{\bullet} \rightarrow Q_{\pi(v)}^{\bullet}$ given by the following unfolded diagram.

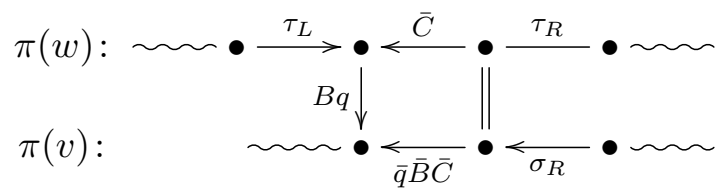

By Remark 1.8, this gives rise to the quasi-graph map $\varphi: Q_{\pi(v)}^{\bullet} \rightsquigarrow Q_{\pi(w)}^{\bullet}$, as claimed. Indeed, one can see that the map given in the unfolded diagram above is one member of the homotopy family determined by $\varphi$. Dual considerations apply for the case $A \neq \varnothing$ and $B^{\bullet}=\varnothing$.

Finally, the case $A=\varnothing$ and $B=\varnothing$ gives rise to a graph map $f^{\bullet}: Q_{\pi(w)}^{\bullet} \rightarrow Q_{\pi(v)}^{\bullet}$, whence a quasi-graph map $\varphi: Q_{\pi(v)}^{\bullet} \rightsquigarrow Q_{\pi(w)}^{\bullet}$ by Remark 1.8. Note that, necessarily, $C \neq \varnothing$ and $D \neq \varnothing$. In this case, by Corollary 2.12, $\pi(v)$ has the form,

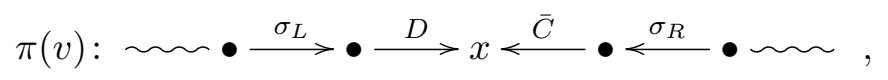

in which the homotopy letters $\sigma_{L}$ and $\sigma_{R}$ may be empty. The homotopy string $\pi(w)$ has one of the following four forms
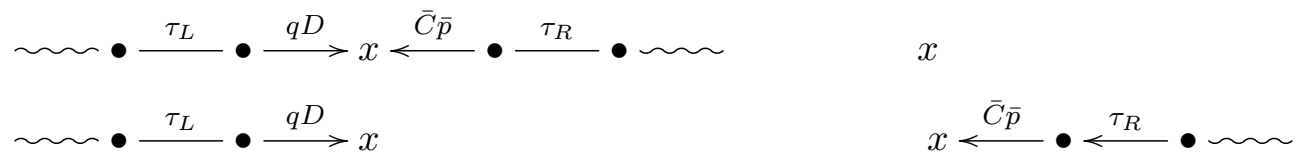

where $p$ and $q$ are (possibly trivial) paths in $(Q, I)$. Whenever $p$ is trivial $\tau_{R} \neq \varnothing$ and is direct; whenever $q$ is trivial $\tau_{L} \neq \varnothing$ and is inverse. The graph map $f^{\bullet}: Q_{\pi(w)}^{\bullet} \rightarrow Q_{\pi(v)}^{\bullet}$ can be read off from the following unfolded diagram, interpreting $p$ and $q$ as trivial paths (whence isomorphisms) and deleting homotopy letters as appropriate to fit the cases.

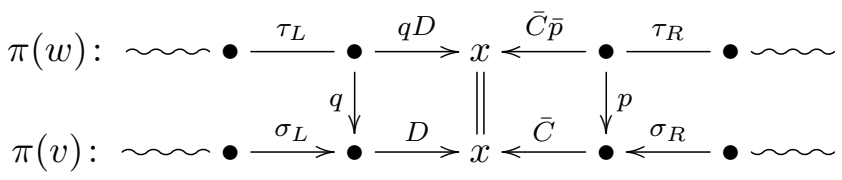

As above, we apply Proposition 3.11 to get $\Phi\left(g^{\bullet}\right)=\eta$.

4.2. Arrow Extensions. Let $v=v_{m} \cdots v_{1}$ and $w=w_{n} \cdots w_{1}$ where $v_{i}, w_{i} \in Q_{1} \cup \bar{Q}_{1}$. Suppose that $\eta \in \operatorname{Ext}_{\Lambda}^{1}(M(v), M(w))$ is an arrow extension corresponding to an arrow $a \in Q_{1}$, i.e. $\eta$ corresponds to an extension with $M(u)$ as the middle term where $u=w a v$.

Since we know $a v$ is defined as a string, then we are in case (1) or (3) in Corollary 2.12 so that $\pi(v)=\operatorname{dir}(a) \widetilde{v}$, where $\widetilde{v}=v^{\prime} \operatorname{inv}(b)$ for some $\bar{b} \in Q_{1}$ or $\widetilde{v}=v^{\prime}$ depending on whether we fall into case (1) or (3), respectively. We set $\operatorname{dir}(a)=\cdots \theta_{2} \theta_{1} a$. Likewise,

$$
\pi(w)= \begin{cases}\widetilde{w} \operatorname{inv}(c) & \text { if there exists } c \in Q_{1} \text { such that } w_{1} \bar{c} \text { is defined as a string; } \\ \widetilde{w} & \text { otherwise, }\end{cases}
$$

where $\widetilde{w}$ is defined in a manner analogous to $\widetilde{v}$, depending on considerations at its end. We write $\operatorname{inv}(c)=\bar{c} \bar{\varphi}_{1} \cdots \bar{\varphi}_{2} \cdots$.

The form of the map $g^{\bullet}: Q_{\pi(v)}^{\bullet} \rightarrow Q_{\pi(w)}^{\bullet}$ such that $\Phi\left(g^{\bullet}\right)=\eta$ depends on whether $v$ ends with an inverse or direct letter and $w$ starts with an inverse or direct letter. We deal with the cases in turn.

Case: $w_{1} \in Q_{1}$ and $v_{m} \in \bar{Q}_{1}$. 
If $\widetilde{w} \operatorname{inv}(c)$ is defined, then we get the unfolded diagram of a (one-sided) graph map, $g^{\bullet}: Q_{\pi(v)}^{\bullet} \rightarrow \Sigma Q_{\pi(w)}^{\bullet}$, below, where we have used $\overline{\pi(w)}$ in the diagram.
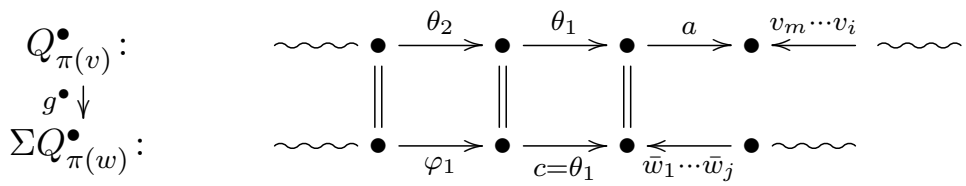

Since $w_{1} a$ is defined as a string, we have $w_{1} a \notin I$, whence $c=\theta_{1}$ by gentleness. Continuing, we see that $\varphi_{i}=\theta_{i+1}$ for each $i>1$. Applying Lemma 3.5 one verifies that $\Phi\left(g^{\bullet}\right)=\eta$.

If $\widetilde{w} \operatorname{inv}(c)$ is not defined, then we get the following unfolded diagram of a (one-sided) graph map $g^{\bullet}: Q_{\pi(v)}^{\bullet} \rightarrow \Sigma Q_{\pi(w)}^{\bullet}$ supported in one degree; applying Lemma 3.5 shows $\Phi\left(g^{\bullet}\right)=\eta$.



Note that since $w_{1} a \notin I$ then $\theta_{1}=\varnothing$ (i.e. $\operatorname{dir}(a)=a$ ) because otherwise $\theta_{1}$ would provide such a $c$ by gentleness of $\Lambda$.

Case: $w_{1} \in Q_{1}$ and $v_{m} \in Q_{1}$.

By the same argument as above, we have one of the following unfolded diagram of a (onesided) graph map, $g^{\bullet}: Q_{\pi(v)}^{\bullet} \rightarrow \Sigma Q_{\pi(w)}^{\bullet}$, depending on whether $\widetilde{w} \operatorname{inv}(c)$ is defined. In both cases, one then applies Lemma 3.5.
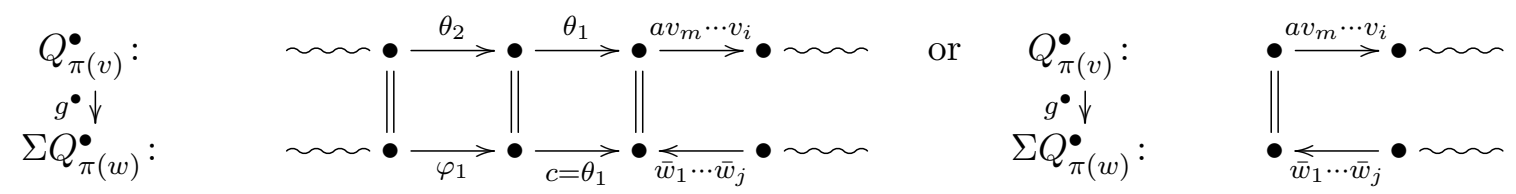

Case: $w_{1} \in \bar{Q}_{1}$ and $v_{m} \in \bar{Q}_{1}$.

Suppose $\widetilde{w} \operatorname{inv}(c)$ is defined. Since $\theta_{1} a \in I$ we have that $\theta_{1} \bar{w}_{1}$ is a string and $c=\theta_{1}$ is the unique arrow such that $c \bar{w}_{1} \notin I$. Continuing we have $\varphi_{i}=\theta_{i+1}$ for $i \geq 1$. This gives the following unfolded diagram of a (one-sided) graph map, $g^{\bullet}: Q_{\pi(v)}^{\bullet} \rightarrow \Sigma Q_{\pi(w)}^{\bullet}$; now apply Lemma 3.5 again.

$$
\begin{gathered}
Q_{\pi(v)}^{\bullet} \\
g^{\bullet} \downarrow \\
\Sigma Q_{\pi(w)}^{\bullet}
\end{gathered}
$$

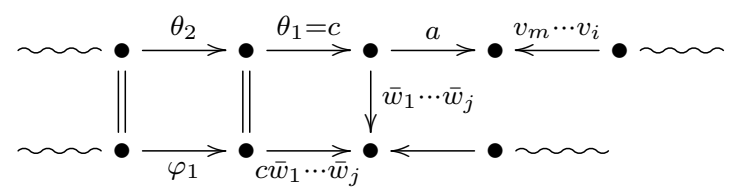

If $\widetilde{w} \operatorname{inv}(c)$ is not defined, then suppose $w_{j} \cdots w_{1}$ is the maximal inverse substring starting $w$, in particular, $\widetilde{w}$ starts with $w_{j+1}$ which is either direct or empty. Furthermore, $\theta_{1}=\varnothing$ for otherwise $w_{1} \bar{\theta}_{1}$ would be defined as a string and we could take $c=\theta_{1}$. Hence we get the following unfolded diagram of a singleton single map $g^{\bullet}: Q_{\pi(v)}^{\bullet} \rightarrow \Sigma Q_{\pi(w)}^{\bullet}$ and we apply Proposition 3.16.

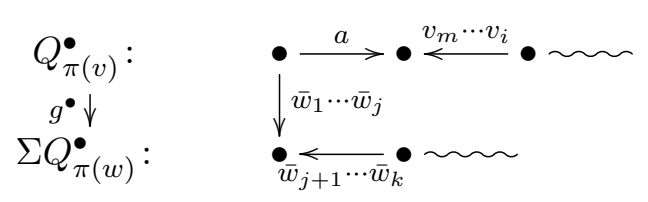

Case: $w_{1} \in \bar{Q}_{1}$ and $v_{m} \in Q_{1}$.

Arguing as above, we get the following unfolded diagram of a (one-sided) graph map or a singleton single map, $g^{\bullet}: Q_{\pi(v)}^{\bullet} \rightarrow \Sigma Q_{\pi(w)}^{\bullet}$, when $\widetilde{w} \operatorname{inv}(c)$ is defined and when it is not, 
respectively. One then applies Lemma 3.5 or Proposition 3.16, respectively.


\section{REFERENCES}

[1] K. K. Arnesen, R. Laking, D Pauksztello, Morphisms between indecomposable complexes in the bounded derived category of a gentle algebra, J. Algebra 467 (2016), 1-46, also arXiv:1411.7644.

[2] I. Assem, T. Brüstle, G. Charbonneau-Jodoin, P.-G. Plamondon, Gentle algebras arising from surface triangulations Algebra Number Theory 4 (2010), no. 2, 201-229, also arXiv:0903.3347/

[3] I. Assem, A. Skowroński, Iterated tilted algebras of type $\widetilde{A}$, Math. Z. 195 (1987), 269-290.

[4] K. Baur, R. Coelho Simões, A geometric model for the module category of a gentle algebra, in press IMRN, https://doi.org/10.1093/imrn/rnz150, also arXiv:1803.05802.

[5] V. Bekkert, H. Merklen, Indecomposables in derived categories of gentle algebras, Algebr. Represent. Theory 6 (2003), 285-302.

[6] R. Bocklandt, Noncommutative mirror symmetry for punctured surfaces. With an appendix by Mohammed Abouzaid. Trans. Amer. Math. Soc. 368 (2016), no. 1, 429-469, also arXiv:1111.3392

[7] T. Brüstle, G. Douville, K. Mousavand, H. Thomas, E. Ylldırım, On the Combinatorics of Gentle Algebras, in press Canadian J. Math. https://doi.org/10.4153/S0008414X19000397, also arXiv:1707.07665.

[8] M. C. R. Butler, C. M. Ringel, Auslander-Reiten sequences with few middle terms and applications to string algebras, Comm. Algebra 15, 145-179.

[9] İ. Çanakçı, D. Pauksztello, S. Schroll, Mapping cones in the bounded derived category of a gentle algebra, J. Algebra 530 (2019), 163-194, also arXiv:1609.09688.

[10] İ. Çanakçi, D. Pauksztello, S. Schroll, Addendum and Erratum: Mapping cones for morphisms involving a band complex in the bounded derived category of a gentle algebra, arXiv:2001.06435.

[11] İ. Çanakçı, S. Schroll, Extensions in Jacobian algebras and cluster categories of marked surfaces, Adv. Math. 313 (2017), 1-49, also arXiv:1408.2074.

[12] W. W. Crawley-Boevey, Maps between representations of zero-relation algebras, J. Algebra 126 (1989), $259-263$

[13] A. Garcia Elsener, Gentle m-Calabi-Yau tilted algebras, arXiv:1701.07968.

[14] F. Haiden, L. Katzarkov, M. Kontsevich, Flat surfaces and stability structures, Publ. Math. Inst. Hautes Études Sci. 126 (2017), 247-318, also arXiv: 1409.8611.

[15] D. Happel, "Triangulated Categories in the Representation Theory of Finite Dimensional Algebras", London Math. Soc. Lecture Notes Series 119, Cambridge University Press (1988).

[16] R. S. Huerfano, M. Khovanov, A category for the adjoint representation, J. Algebra 246 (2001), no. 2, 514-542, also arXiv:math/0002060.

[17] B. Huisgen-Zimmermann, S. O. Smalø, The homology of string algebras. I, J. Reine Angew. Math. 580 (2005), 1-37.

[18] M. Kalck, Singularity categories of gentle algebras, Bull. Lond. Math. Soc. 47 (2015), no. 1, 65-74, also arXiv: 1207.6941.

[19] H. Krause, Maps between tree and band modules, J. Algebra 137 (1991), 186-194.

[20] D. Labardini-Fragoso, Quivers with potentials associated to triangulated surfaces, Proc. Lond. Math. Soc. (3) 98 (2009), no. 3, 797-839, also arXiv:0803.1328.

[21] Y. Lekili, A. Polishchuk, Derived equivalences of gentle algebras via Fukaya categories, in press Math. Annalen, https://doi.org/10.1007/s00208-019-01894-5, also arXiv:1801.06370.

[22] T. McConville, Lattice structure of Grid-Tamari orders, J. Combin. Theory Ser. A 148 (2017) 27-56, also arXiv: 1504.05213.

[23] S. Opper, P.-G. Plamondon, S. Schroll, A geometric model for the derived category of gentle algebras, arXiv: 1801.09659.

[24] Y. Palu, V. Pilaud, P.-G. Plamondon, Non-kissing complexes and $\tau$ tilting for gentle algebras, to appear Mem. Amer. Math. Soc., arXiv:1707.07574.

[25] J. Schröer, Modules without self-extensions over gentle algebras, J. Algebra 216 (1999), no. 1, 178-189. 
[26] D. Simson, A. Skowroński, Elements of the representation theory of associative algebras. Vol. 3. Representation-infinite tilted algebras, London Mathematical Society Student Texts, 72. Cambridge University Press, Cambridge, 2007.

[27] D. Vossieck, The algebras with discrete derived category, J. Algebra 243 (2001) 168-176.

[28] B. Wald, J. Waschbüsch, Tame biserial algebras, J. Algebra 95 (1985), 480-500.

[29] J. Zhang, On the indecomposable exceptional modules over gentle algebras, Comm. Alg. 42 (2014), 3096-3199.

Department of Mathematics, VU Amsterdam, Amsterdam $1081 \mathrm{HV}$, The Netherlands.

E-mail address: i.canakci@vu.nl

Department of Mathematics and Statistics, Lancaster University, Lancaster, LA1 4YF, United KINGDOM

E-mail address: d.pauksztello@lancaster.ac.uk

Department of Mathematics, University of Leicester, University Road, Leicester, LE1 7RH, UNITED KingDOM.

E-mail address: schroll@leicester.ac.uk 